\title{
NSRL Extraction Bump Control in the Booster
}

\author{
L. Brennan
}

Summer 2008

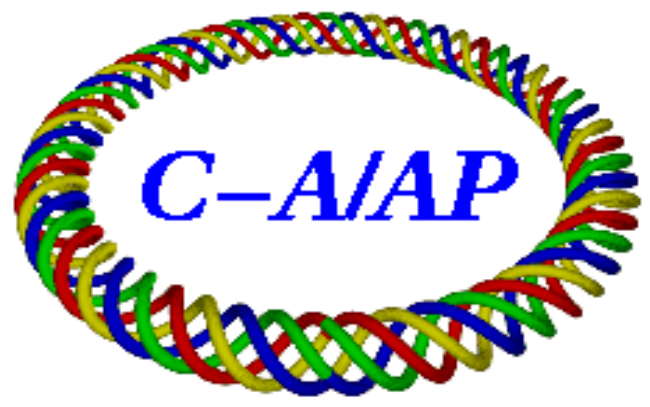

Collider-Accelerator Department Brookhaven National Laboratory

Upton, NY 11973

Notice: This document has been authorized by employees of Brookhaven Science Associates, LLC under Contract No. DE-AC02-98CH10886 with the U.S. Department of Energy. The United States Government retains a nonexclusive, paid-up, irrevocable, world-wide license to publish or reproduce the published form of this document, or allow others to do so, for United States Government purposes. 


\title{
NSRL Extraction Bump Control in the Booster
}

\author{
L. Brennan \\ Summer 2008
}

\begin{abstract}
Due to inadequacies in the user interface of the booster orbit control system, a number of new tools were developed. The first priority was an accurate calculation of the winding currents given specific displacements at each extraction septa. Next, the physical limits of the power supplies ( $\pm 600 \mathrm{amps})$ needed to be taken into account. In light of this limit, a system is developed that indicates to the user what the allowed values of one bump parameter are once the other two have been specified. Finally, techniques are developed to account for the orbit behavior once power supplies are requested to exceed their $\pm 600 \mathrm{amp}$ limit. This includes a recalculation of bump parameters and a calculation of the amplitude of the residuals. Following this, possible areas for further development are outlined. These techniques were computationally developed in Mathematica and tested in the Methodical Accelerator Design (MAD) program before they were implemented into the control system. At the end, a description of the implementation of these techniques in a new interface is described. This includes a depiction of the appearance and functionality of the graphical user interface, a description of the input and output flow, and an outline of how each important calculation is performed.
\end{abstract}

\section{Introduction}

Slow extraction from the Booster is accomplished through a resonant extraction procedure using two septa: a thin electric septum at D3 and a thick magnetic septum at $\mathrm{D} 6^{1}$. Part of this procedure involves a local orbit distortion (bump) created using one extra trim winding set on each of five main dipole magnets centered about the D3 and D6 septa. This winding allow a user to specify the transverse beam positions at D3 and D6 as well as the trajectory angle at one of the two positions.

The purpose of the control system is to allow a user to specify parameters for the bump and to calculate the currents required in the trim windings to achieve these parameters. However, not all configurations are possible due to a physical limit of \pm 600 amps of the power supplies used. When a current is requested that exceeds the range of the power supplies, they are set to their maximum, resulting in various undesirable behaviors. Currently, the system calculates the necessary currents and indicates to a user if the requested configuration is not possible, but it does not give the user guidance regarding allowed configurations, and it does not account for the behavior of the orbit once the power supply limits have been exceeded.

In order to improve this control system, several techniques needed to be developed. First, a method of calculating the allowed range of one parameter, once the others had been set, was devised. Second, the effects of the limits of the power supplies were accounted for. This meant calculating the bump parameters that the new

\footnotetext{
${ }^{1}$ For more information on NSRL extraction in the AGS Booster see L. Hammons et al. Study of Orbits Produced by Individual Booster Extraction Windings for the NASA Space Radiation Laboratory, C-A/AP/\#282. July 2007.
} 
configuration of currents would achieve. It also meant developing a method of estimating the amplitude of orbit distortions outside the bump, known as residuals.

This paper first outlines the basic physics and mathematics of orbit distortion. It then develops the content of the control system, beginning with the calculation of the necessary currents, followed by the calculation of allowed ranges, and ends with the effects of the limit of the power supplies. On several occasions, the routines developed are demonstrated using Mathematica ${ }^{2}$ and tested using the Methodical Accelerator Design (MAD) program ${ }^{3}$. Following this development, an outline of the implementation is given, including the graphical user interface and the details of each calculation.

\section{General Bumps}

Let us begin our discussion with an outline of the basic theory behind local orbit distortions ${ }^{4}$, bumps. A bump is composed of a sequence of kicks, such that outside of the bump, the transverse displacement returns to zero. These kicks are given by extra windings added to the main dipole magnets and so can be understood as errors in the strength of the main dipole field. Any errors in the magnets will result in a new closed orbit solution, which can be expressed in relation to the reference orbit. Considering an ideal particle traveling along the reference orbit, we can see that after passing through one error, the particle has received a kick of angle $\theta$ and a change in transverse trajectory, $\Delta x^{\prime}$, given:

$$
\begin{gathered}
\theta=\Delta x^{\prime}=\frac{\Delta s}{\Delta \rho} \\
\theta=\Delta x^{\prime}=-\frac{e \Delta s \Delta B}{p}=-\frac{\Delta s \Delta B}{B \rho}
\end{gathered}
$$

where $\Delta \mathrm{s}$ stands for the azimuthal length of the field error, $\rho$ stands for the radius of curvature of the field error, $\Delta B$ stands for the magnitude of the field error, $e$ stands for the charge of the particle, $p$ stands for its momentum, and $B \rho$ is magnetic rigidity. Here we take the definition of radians in equation (1) and substitute $\Delta B / B \rho=-1 / \Delta \rho$ to get equation (2). The negative sign here (seen in equation (2)) has been added to note that an increase in magnetic field will decrease the radius of curvature. At the point of the error, $\mathrm{x}$ ' $=\Delta \mathrm{x}$ ' $/ \Delta \mathrm{s}$ will be equal to $\Delta \mathrm{B} / \mathrm{B} \rho$. Assuming we are dealing with a strong focusing synchrotron, we already have an expression for $\mathrm{x}$ ", according to the focusing function $\mathrm{K}(\mathrm{s})$ and neglecting higher order fields (those higher than quadrupole). Adding this new expression to the one already known we discover that:

\footnotetext{
${ }^{2}$ Wolfram Research, Inc.

${ }^{3}$ MAD-X, CERN Accelerator Physics Group

${ }^{4}$ For more information regarding field deformations see M. Sands, The Physics of Electron Storage Rings, SLAC report 121, 1970, esp. pgs.49-53. For more information regarding bumps, see A. Luccio, Bumps in the AGS Booster: Booster Technical Note No. 189. 1991.
} 


$$
x^{\prime \prime}=K(s) x+\frac{\Delta B}{B \rho}
$$

Assuming no other field errors, this looks very similar to the equation of betatron oscillation with a driving term $\Delta \mathrm{B} / \mathrm{B} \rho$. Taking the error to be placed at $\mathrm{s}_{0}$ the general form of these oscillations is:

$$
x(s)=a \sqrt{\beta(s)} \cos \left(\left|\phi(s)-\phi\left(s_{o}\right)\right|-\psi\right)
$$

where $\beta$ and $\phi$ are the beta function and the phase advance defined in the Courant-Snyder parameters respectively.

Since we aim to find the new closed orbit, $\mathrm{x}_{\mathfrak{c}}$, we can impose the following boundary conditions: that the displacement, $\mathrm{x}$, is periodic with respect to the accelerator circumference, $\mathrm{L}$, and that the trajectory angle before the error plus the error equals the trajectory angle after the error. Equivalently:

$$
\begin{gathered}
x_{c}(s)=x_{c}(s+L) \\
x_{c}^{\prime}\left(s_{o}+L\right)+\Delta x^{\prime}=x_{c}^{\prime}\left(s_{o}\right)
\end{gathered}
$$

From the first boundary condition, we can solve for the phase factor $\psi$ and from the second condition we can find $a$. These are:

$$
\begin{gathered}
\psi=\pi v \\
a=\frac{\theta \sqrt{\beta\left(s_{o}\right)}}{2 \sin (\pi v)}
\end{gathered}
$$

where $v$ is the betatron tune. Hence, the new, disturbed closed orbit that will result from this field error will be:

$$
x_{c}(s)=\frac{\sqrt{\beta(s)}}{2 \sin (\pi v)} \theta \sqrt{\beta\left(s_{o}\right)} \cos \left(\left|\phi(s)-\phi\left(s_{o}\right)\right|-\pi v\right)
$$

The question now becomes: what happens with multiple errors as in a bump? To see this, we return to the original differential equation (3) and note that we have disregarded non-linear terms in $\mathrm{x}$. This leaves us with a linear equation for each error, which then can be linearly combined:

$$
\left(\sum x_{i}\right)^{\prime \prime}=K(s) \sum x_{i}+\frac{e}{p} \sum \Delta B_{i}
$$

So, the closed orbit solution for $\mathrm{n}$ field errors, each at $\mathrm{s}_{i}$, will be the linear combination of the orbits for each error. 


$$
x_{c}(s)=\frac{\sqrt{\beta(s)}}{2 \sin (\pi v)} \sum_{i=1}^{n} \sqrt{\beta\left(s_{i}\right)} \theta_{i} \cos \left(\left|\phi(s)-\phi\left(s_{i}\right)\right|-\pi v\right)
$$

To find the angle at $\mathrm{s}$, we simply take the derivative, introducing $\alpha(\mathrm{s})$ as one of the Courant-Snyder parameters:

$$
\begin{gathered}
x_{c}^{\prime}(s)=-\frac{\alpha(s)}{\beta(s)} x_{c}(s)-\frac{1}{2 \sin (\pi v) \sqrt{\beta(s)}} \sum_{i=1}^{n} \sqrt{\beta\left(s_{i}\right)} \theta_{i} \sin \left(\left|\phi(s)-\phi\left(s_{i}\right)\right|-\pi v\right) \\
\alpha(s)=-\frac{\beta^{\prime}(s)}{2}
\end{gathered}
$$

In a bump, we would like to specify the $\mathrm{x}$ position at several points. To do this generally, because of the linearity, we can conveniently write the following matrix equation:

$$
\left(\begin{array}{c}
x_{1} \\
\vdots \\
x_{n}
\end{array}\right)=\left(\begin{array}{ll}
A \\
\end{array}\right)\left(\begin{array}{c}
\theta_{1} \\
\vdots \\
\theta_{n}
\end{array}\right)
$$

where:

$$
x\left(s_{j}\right)=x_{j}
$$

and the entries of the matrix are such that the following is equivalent to equation (5) or (6) depending on whether $\mathrm{x}_{\mathrm{j}}$ denotes a displacement or an angle since either are possible:

$$
x_{j}=\sum_{i} A_{j i} \theta_{i}
$$

Since we would like to specify offsets and angles and can control kicks (through current in the windings), we solve equation (7) by finding $\mathrm{A}^{-1}$ :

$$
\begin{gathered}
\left(\begin{array}{c}
\theta_{1} \\
\vdots \\
\theta_{n}
\end{array}\right)=\left(\begin{array}{c}
A^{-1} \\
\end{array}\right)\left(\begin{array}{c}
x_{1} \\
\vdots \\
x_{n}
\end{array}\right) \\
x_{j}=\sum_{i} A_{j i}^{-1} \theta_{i}
\end{gathered}
$$

We close the bump by setting $\mathrm{x}=0$ and $\mathrm{x}^{\prime}=0$ at the final kick (as in equation (9)). In the case of the extraction 5-bump in the Booster that is being discussed we can more specifically say that: 


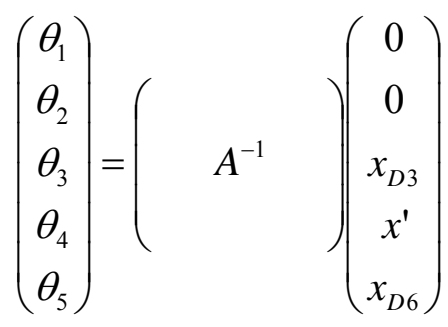

Here, the first two values in the displacement vector are set at zero corresponding to the values of $x$ and $x$ ' at position E1, the final kick in the bump. The fourth entry in the displacement vector, denoted x', can either be x' at D3 or at D6 depending on the entries in the fourth row of $\mathrm{A}$.

\section{Kicks to Currents}

At this point we have a way to find the necessary kick angles given certain bump parameters. For a control system, these kicks must now be turned into currents that can be sent through the trim windings. Looking at equation (2), we can see that this boils down to finding the relationship between the change in B-field needed and the current in the bump coils. Once one considers non-linear effects in the magnets, like saturation, this ceases to be straightforward.

The change in B-field created by the winding can be expressed as the difference between the main dipole field and the field produced by the total current from both the main dipole windings and the additional bump coils:

$$
\Delta B=B\left(I_{\text {total }}\right)-B\left(I_{\text {dipole }}\right)
$$

Since the main dipole windings are composed of two coils of eight turns each and the bump coils have two turns, the total current is:

$$
I_{\text {total }}=I_{\text {main }}+\frac{2}{16} I_{\text {Winding }}
$$

which implies that:

$$
\frac{16}{2}\left[B^{-1}\left(\Delta B+B\left(I_{\text {main }}\right)\right)-I_{\text {main }}\right]=I_{\text {Winding }}
$$

The function $\mathrm{B}(\mathrm{I})$ is the function relating the magnetic field to the current in the windings and $\mathrm{B}^{-1}$ is its inverse. A typical expansion of $\mathrm{B}(\mathrm{I})$ used in the Booster has been developed by Kevin Brown:

$$
\begin{aligned}
\mathrm{B}(\mathrm{i})= & 0.0009122+0.0002371 \mathrm{i}+1.717 \times 10^{-8} \mathrm{i}^{2}-2.412 \times 10^{-11} \mathrm{i}^{3}+1.836 \times 10^{-14} \mathrm{i}^{4} \\
& -7.88 \times 10^{-18} \mathrm{i}^{5}+1.891 \times 10^{-21} \mathrm{i}^{6}-2.351 \times 10^{-25} \mathrm{i}^{7}+1.163 \times 10^{-29} \mathrm{i}^{8}
\end{aligned}
$$


There are a few strategies that can be used to find $\mathrm{B}^{-1}$ from this expression. Either one can plot data points of an inverse using this function, i.e. calculate a set of points $(B(i), i)$, and fit a new curve to it; or one can use a solution algorithm to numerically find the current given the B-field. A numerical method can lead to multiple and erroneous solutions and in this case, a fitted inverse is more useful.

A fitted inverse has been developed in Mathematica. First, a list of ordered pairs $(\mathrm{B}[\mathrm{i}], \mathrm{i})$ is created, where, again, "bfld" represents equation (12) and "i" is a current varying from 0 to 5000 amps, a suitable increment for "i" should be 250 amps though smaller increments will yield higher accuracy. The syntax for this is as follows:

$$
\text { list }=\text { Table }[\{b f l d[i], i\},\{i, 0,5000,250\}]
$$

Next, this list is used as data for calculating a polynomial fit. Mathematica can find a $20^{\text {th }}$ order polynomial that is accurate out to machine precision, but this is computationally expensive and offers a non-valuable gain in accuracy. Instead, the "FindFit" function was used to calculate coefficients for an eighth-order polynomial. The Mathematica input as well as the resulting polynomial are shown below:

$$
\begin{aligned}
& \text { binv }\left[\mathrm{x}_{-}\right]:= \\
& \mathrm{a}+\mathrm{b} \mathrm{x}+\mathrm{c} \mathrm{x}^{\wedge} 2+\mathrm{d} \mathrm{x}^{\wedge} 3+\mathrm{e} \mathrm{x}^{\wedge} 4+\mathrm{f} \mathrm{x}^{\wedge} 5+\mathrm{g} \mathrm{x}^{\wedge} 6 \text { th } \mathrm{x}^{\wedge} 7+i \mathrm{x}^{\wedge} 8 \\
& \text { /.FindFit [list, } \\
& \mathrm{a}+\mathrm{b} \mathrm{x}+\mathrm{c} \mathrm{x}^{\wedge} 2+\mathrm{d} \mathrm{x}^{\wedge} 3+\mathrm{e} \mathrm{x}^{\wedge} 4+\mathrm{f} \mathrm{x}^{\wedge} 5+\mathrm{g} \mathrm{x}^{\wedge} 6+\mathrm{h} \mathrm{x}^{\wedge} 7+\mathrm{i} \mathrm{x}^{\wedge} 8 \text {, } \\
& \{a, b, c, d, e, f, g, h, i\},\{x\}] \\
& \mathrm{B}^{-1}(\mathrm{x})=-3.74879+4190.08 \mathrm{x}-643.808 \mathrm{x}^{2}+2578.06 \mathrm{x}^{3}-5126.86 \mathrm{x}^{4}+4101.54 \mathrm{x}^{5} \\
& +1312 . \mathrm{x}^{6}-3992.35 \mathrm{x}^{7}+1727.21 \mathrm{x}^{8}
\end{aligned}
$$

This expansion is accurate within a tenth of a percent up to 1.2 Tesla, the field associated with 5170 amps and above the normal operating levels of the main dipole magnets. The $20^{\text {th }}$ order polynomial is accurate within a thousandth of a percent at 1.2 Tesla. Since, a tenth of a percent is more than sufficient, only the eighth order polynomial will be used. We can graph the pure numerically evaluated inverse of B with the two expansions to see the general trend of divergence (Figure 1). As we can see, the three are close within the convergent region of the original expansion of $\mathrm{B}$, which is approximately up to 5500 amps. In the absence of Mathematica, equivalent expansions to those described above can easily be found using least square algorithms to find coefficients. 


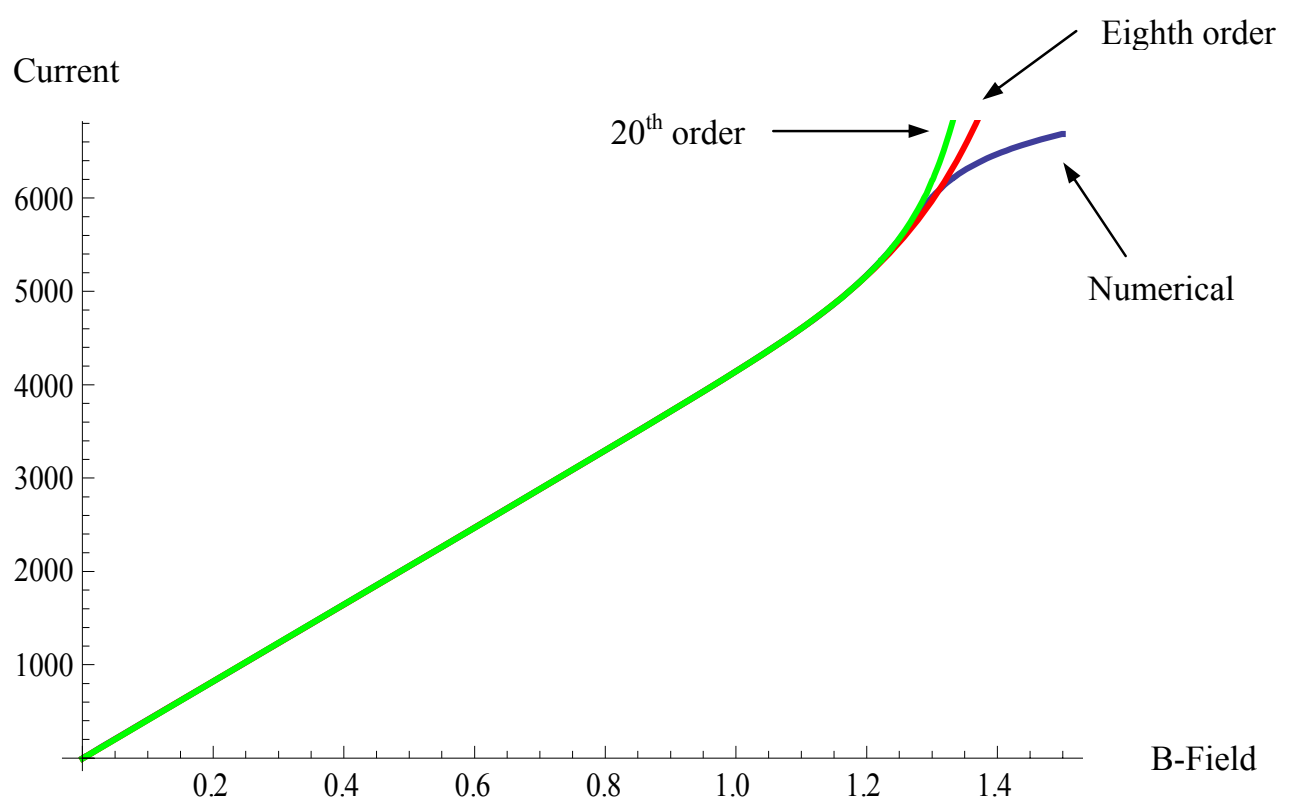

Figure 1. Three approximations for current as a function of magnetic field.

In order to evaluate the accuracy of these calculations, the results were tested using MAD. These simulations were conducted using the Booster lattice maintained by Kevin Brown and equation (12) above for the expansion of the magnetic field in the main dipoles. Below is a plot of the $\mathrm{x}$ displacement of the orbit with respect to the $\mathrm{s}$ coordinate. Here, the trim winding currents calculated for a main dipole current of 4723 amps, $3 \mathrm{~mm}$ offsets at D3 and D6, and an angle of $1 \mathrm{mrad}$ at D6, were input into MAD.

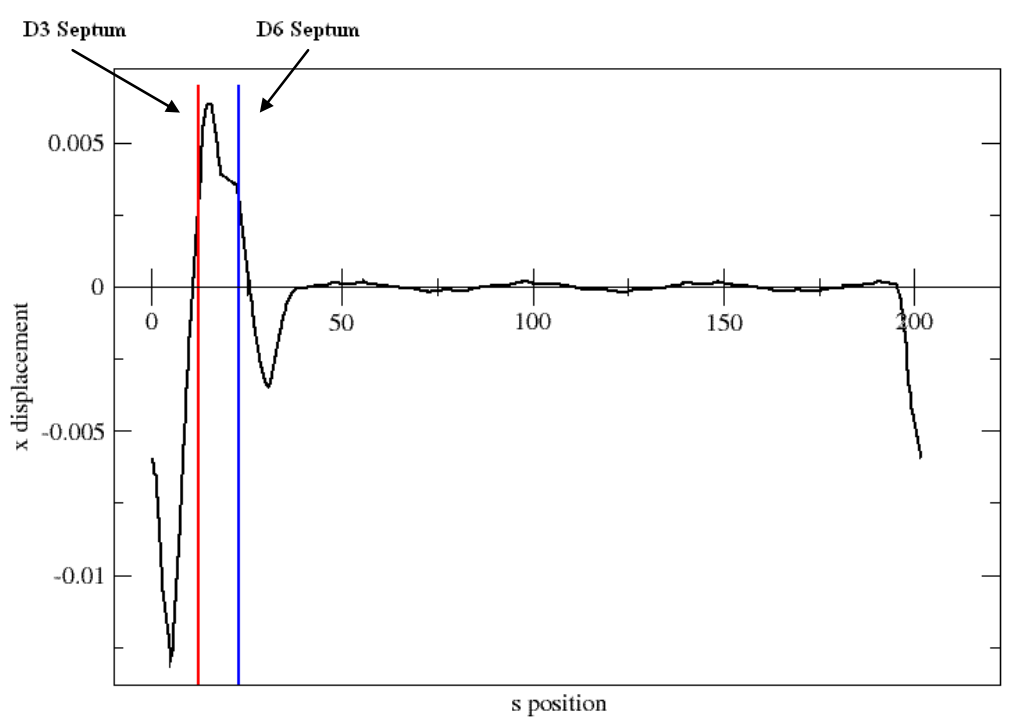

Figure 2. Horizontal transverse orbit generated by MAD.

Main dipole field at 4723 and offsets set to $3 \mathrm{~mm}$ at each septum and the angle at D6 set to $-1 \mathrm{mrad}$.

The offsets calculated by MAD were $3.1 \mathrm{~mm}$ at D3, $2.96 \mathrm{~mm}$ at D6, and an angle of $-1 \mathrm{mrad}$ at D6. This agrees strongly with the desired values. To demonstrate the 
accuracy of the calculations over a range of settings, let us examine two more MAD simulations.

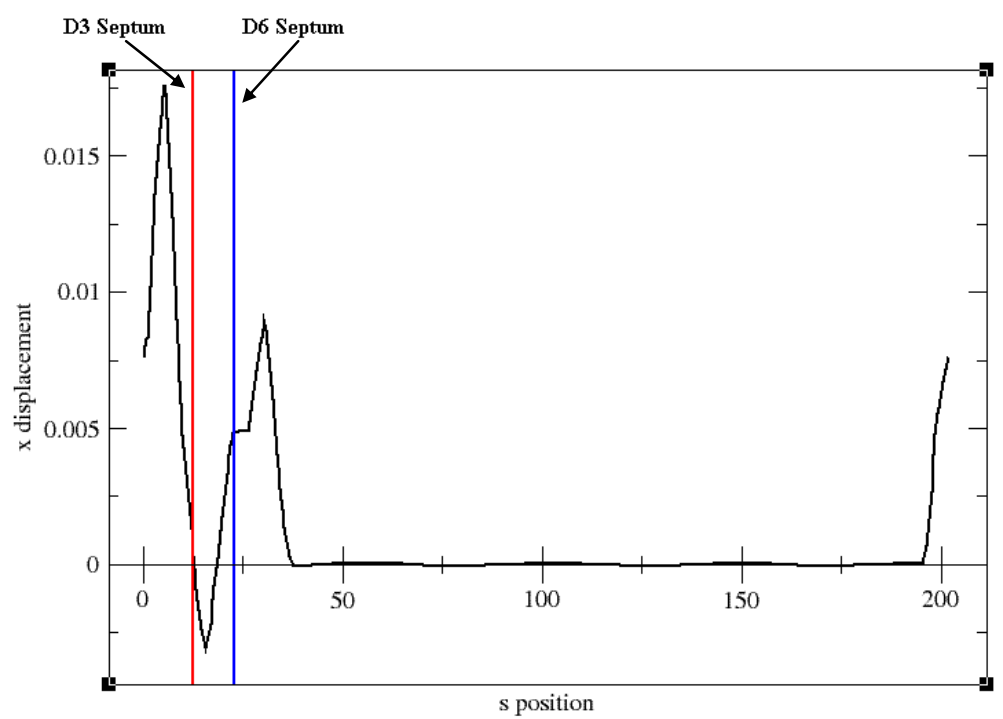

Figure 3. Horizontal transverse orbit generated by MAD.

Main dipole field at 4775, D3 offset at $1 \mathrm{~mm}$, D6 offset at $5 \mathrm{~mm}$ and the angle at D6 set to $0.0 \mathrm{mrad}$.

In Figure 3, the main dipole current is set to 4775 amps and an offset of $1 \mathrm{~mm}$ at D3 and $5 \mathrm{~mm}$ at D6 is requested, along with an angle at D6 of $0.0 \mathrm{mrad}$. The calculated values shown above were as follows: offset at D3 $=0.076 \mathrm{~mm}$, offset at D6 $=4.87 \mathrm{~mm}$, and D6 angle $=0.01$ mrad.

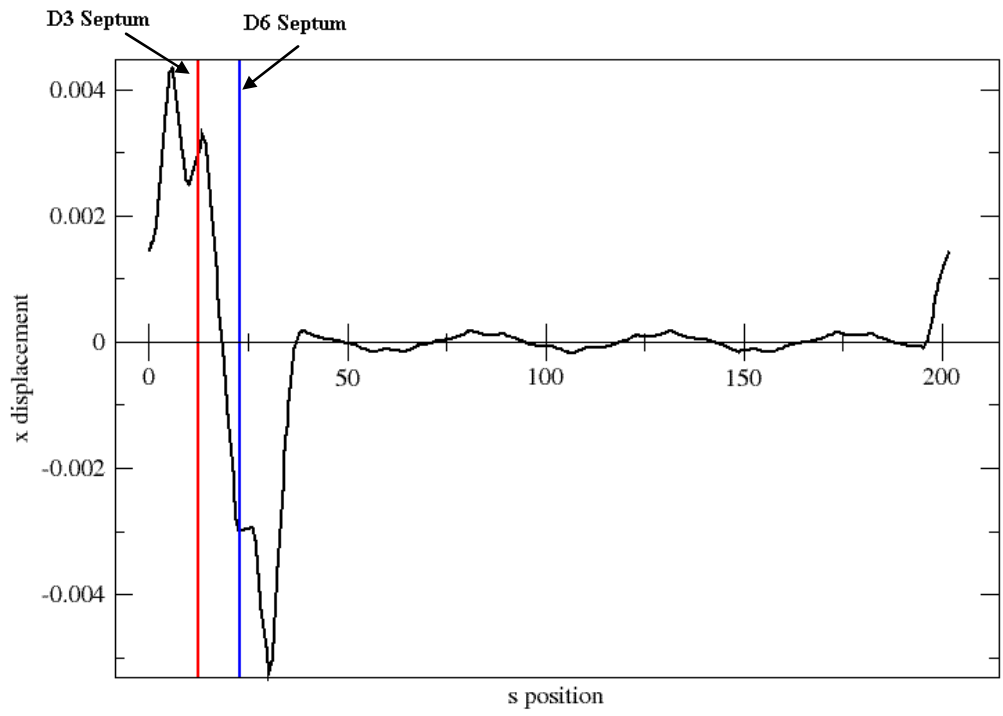

Figure 4. Horizontal transverse orbit generated by MAD.

Main dipole field at 4775, D3 offset at $3 \mathrm{~mm}$, D6 offset at $-3 \mathrm{~mm}$ and the angle at D6 set to $0.0 \mathrm{mrad}$.

In Figure 4, an offset of $3 \mathrm{~mm}$ at D3, $-3 \mathrm{~mm}$ at D6, and an angle of $0.0 \mathrm{mrad}$ at D6 were requested. The simulation run by MAD calculated an offset of $3 \mathrm{~mm}$ at D3, 
$-2.98 \mathrm{~mm}$ at D6, and an angle of $0.01 \mathrm{mrad}$ at D6. In each of the above figures, it should be noted that the residuals are on the order of $0.1 \mathrm{~mm}$ or lower.

\section{Calculating the Range}

As mentioned above the power supplies for the bump windings have a limit of \pm 600 amps. This means that many of the combinations of offsets and trajectories requested by the user will not be possible. For example, something as simple as a $3 \mathrm{~mm}$ offset at D3 and D6 with a 3 mrad value of x' at D6 in a main dipole current of 4723 amps would require -3670.38 amps in the $\mathrm{C} 7$ winding and would exceed the limit for each of the other power supplies as well. In the old interface, the user who requested this combination would have no way of knowing that once they specified the offsets of three millimeters at both D3 and D6 they restricted their range of allowed values of x' at D6 to between $-1.12189 \mathrm{mrad}$ and $-.0443433 \mathrm{mrad}$. The ability to inform the user of this range, the allowed range of one parameter once two others are set, is an important addition in the new interface and is developed below.

To find this range analytically, we can start with the relationship between one kick angle $\theta_{\mathrm{i}}$ to the specified displacements at D3 and D6 and the angle at one of these two locations. From equation (8) we find that:

$$
\theta_{i}=A_{i 3}^{-1} x_{3}+A_{i 4}^{-1} x_{4}+A_{i 5}^{-1} x_{5}
$$

where $\mathrm{x}_{3}=\mathrm{x}_{\mathrm{D} 3}, \mathrm{x}_{4}=\mathrm{x}^{\prime}$ at either D3 or D6 and $\mathrm{x}_{5}=\mathrm{x}_{\mathrm{D} 6}$. This, by equation (2), implies that:

$$
-\frac{\Delta B_{i} \Delta s}{B \rho}=A_{i 3}^{-1} x_{3}+A_{i 4}^{-1} x_{4}+A_{i 5}^{-1} x_{5}
$$

where $\Delta \mathrm{B}_{\mathrm{i}}$ is the change in magnetic field at the $\mathrm{i}^{\text {th }}$ winding. From this, we can find four sets of equations, one relating current to all three parameters and three relating each of the offset parameters to current as well as the other two parameters:

$$
\begin{gathered}
I_{i}=B^{-1}\left[B\left(I_{\text {main }}\right)-\frac{B \rho}{\Delta s}\left(A_{i 3}^{-1} x_{3}+A_{i 4}^{-1} x_{4}+A_{i 5}^{-1} x_{5}\right)\right]-I_{\text {main }} \\
x_{4}=-\frac{1}{A_{i 4}^{-1}}\left(\frac{\Delta s \Delta B_{i}}{B \rho}+A_{i 3}^{-1} x_{3}+A_{i 5}^{-1} x_{5}\right) \\
x_{3}=-\frac{1}{A_{i 3}^{-1}}\left(\frac{\Delta s \Delta B_{i}}{B \rho}+A_{i 4}^{-1} x_{4}+A_{i 5}^{-1} x_{5}\right) \\
x_{5}=-\frac{1}{A_{i 5}^{-1}}\left(\frac{\Delta s \Delta B_{i}}{B \rho}+A_{i 4}^{-1} x_{4}+A_{i 3}^{-1} x_{3}\right)
\end{gathered}
$$

Each of the above equations represents five different equations, indexed by " $i$ ", one for each winding. There are two things one should take note of here: first, that the five equations are not independent since each depends on all three offset parameters. 
Equation (14) is an explicit statement of the matrix equation (9) and the calculation of the currents described above. Equations (15), (16), and (17) are all then slightly confusing. The five equations they represent cannot be treated independently if one desires physical results. With the same offsets, $x_{3}$ and $x_{5}$ set in equation (15) one can calculate five different angles by using different currents in each winding, which is not physical. One must not forget that the offsets are also functions of the current in each winding. If one finds an angle using (15) at two offsets and a current in one winding, the current in each of the other windings is uniquely determined by equation (14). This confusion may seem to make these equations unhelpful. However, if we take them simply to be solutions of equation (14), they are vital to finding the allowed range as will be seen below.

Second, it should be noted that while we cannot assume the linearity of B, we can assume that it is monotonically increasing within the range we are working. This means that in determining the relationship between $\mathrm{I}_{\mathrm{i}}$ and one offset parameter (so long as we care only about sign), it is sufficient to determine the relationship between that parameter and the argument of B. This can be seen more clearly from the derivative, with $\mathrm{j}=3,4,5$ :

$$
\frac{\partial}{\partial x_{j}} I_{i}=\frac{\partial}{\partial x_{j}} B^{-1}\left[B\left(I_{\text {main }}\right)-\frac{B \rho}{\Delta s}\left(A_{i 3}^{-1} x_{3}+A_{i 4}^{-1} x_{4}+A_{i 5}^{-1} x_{5}\right)\right]\left(\frac{-B \rho A_{i j}^{-1}}{\Delta s}\right)
$$

Since $\mathrm{B} \rho$ and $\Delta \mathrm{s}$ are both positive and $\mathrm{B}$ is monotonically increasing, the sign of the derivative of $I_{i}$ with respect to a certain parameter depends only on the sign of the matrix element $A_{i j}{ }^{-1}$ where " $i$ " specifies the winding and " $j$ " specifies the offset parameter. Explicitly, the sign of the derivative will be opposite the sign of $\mathrm{A}_{\mathrm{ij}}{ }^{-1}$.

For a clearer illustration of this, we can graph the five winding currents as one parameter is changed and the other two held constant. For example, setting $x[D 3]=3$ $\mathrm{mm}, \mathrm{x}[\mathrm{D} 6]=-4 \mathrm{~mm}$, a main dipole current of 4775 amps, varying the trajectory angle at D6 and plotting the current in each winding according to equation (15), we obtain the following graph:

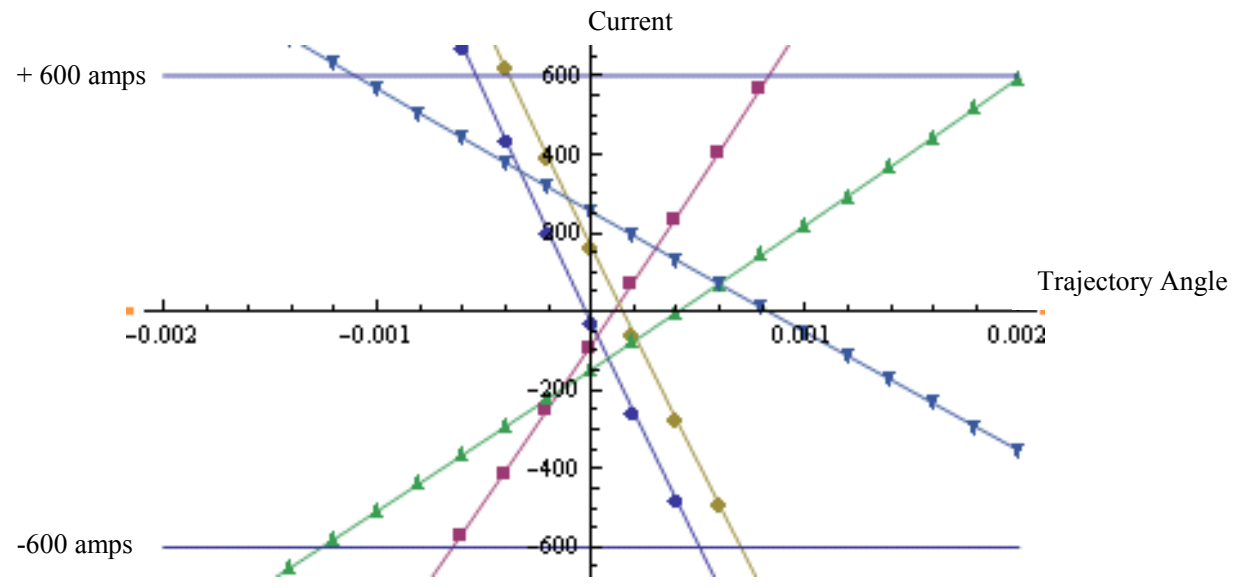

Figure 5. Currents varying with trajectory angle.

$3 \mathrm{~mm}$ offset at D3 and $-4 \mathrm{~mm}$ offset at D6. Markings indicate the following: IC7, circles; ID1, squares; ID4, diamonds; ID7, upward pointing triangles; IE1, downward facing triangles.

In this graph the line marked by circles is $\mathrm{C}$, the line marked by squares is D1, the line marked by diamonds is $\mathrm{D} 4$, the line marked by upward pointing triangles is $\mathrm{D} 7$ 
and the line marked by downward pointing triangles is E1. The range of allowed values will be the range in which none of the lines above exceed 600 amps. An example of when this region will be empty is for an offset of 10 millimeters at D3 and -16 millimeters at D6:

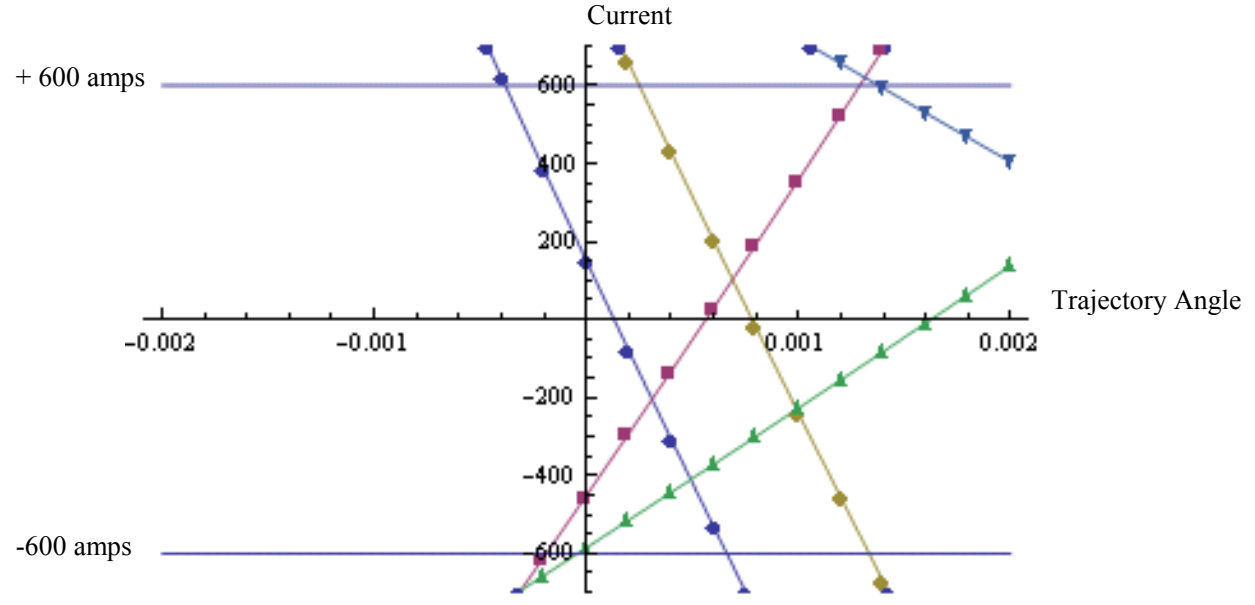

Figure 6. Currents varying with trajectory angle.

10mm offset at D3 and $-16 \mathrm{~mm}$ offset at D6. Markings indicate the following: IC7, circles; ID1, squares;

ID4, diamonds; ID7, upward pointing triangles; IE1, downward facing triangles.

Each line has an allowed range, and, as we can see, the general allowed range will be the intersection of all the allowed ranges. Let us focus on one winding in Figure 5 and develop a method for finding the range of allowed trajectory angles. One winding is equivalent to one line in Figure 5 and one equation from the five in (14). Below is the plot for the current in D1 as the angle varies.

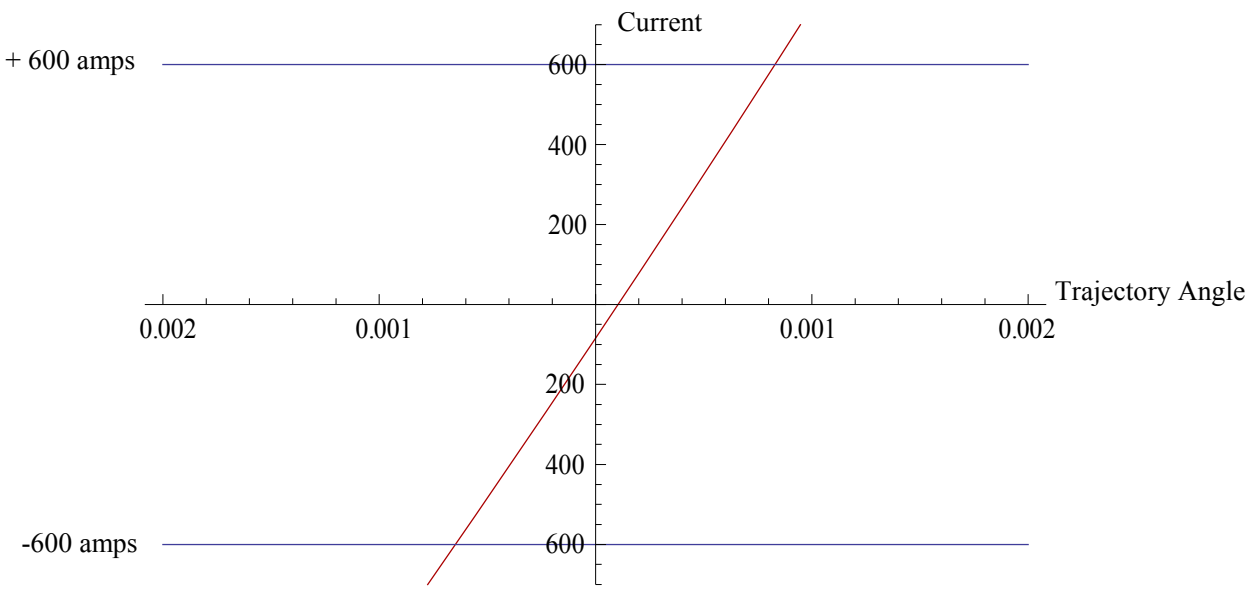

Figure 7. Current of D1 varying with trajectory angle. $3 \mathrm{~mm}$ offset at D3, -4mm offset at D6 and main dipole current of 4775 .

Here the allowed range of this winding is from where the line intersects -600 amps to where it intersects +600 amps. In general, each line has two intersections with \pm 600 amps, one with +600 and one with -600 . These two intersections will be associated with a single trajectory angle. Since these lines are monotonic, the angles have a simple relationship to the allowed range for this power supply. For all trajectory angles between the angle associated with the left limit (the left limiting angle) and the angle associated with the right limit (the right limiting angle) D1 will be within its allowed range. Since 
the line representing D1 happens to be positive slope the left limiting angle occurs at -600 amps and the right limiting angle occurs at +600 amps (this can be easily verified from the plot above). This relationship will be the same for all lines of positive slope. For lines of negative slope however the left limiting angle will be associated with the +600 amp limit and the right limiting angle will occur at -600 amps.

This gives us a way to find the allowed range for each power supply. First, we test the slope of the line, and then, we find the left limiting angle and the right limiting angle. If the slope is positive we set the current equal to -600 amps in equation (14) and solve for the left limiting angle, and we set the current equal to +600 amps in the equation (14) and solve for the right limiting angle, if the slope is negative we do the reverse. As shown by equation (18), the sign of the slope of the line depends only on the matrix element $\mathrm{A}_{\mathrm{i} 4}{ }^{-1}$. The job of setting the current equal to \pm 600 amps in (14) and solving for the angle is equivalent to setting the current equal to $\pm 600 \mathrm{amps}$ in equation (15).

We can now turn to calculating the general allowed range as the intersection of all these allowed ranges. Since each range is independent, the general allowed range will be from one of the five left limiting angles to one of the five right limiting angles. More specifically, since our currents change monotonically, the general left limiting angle will be the maximum of the five left limiting angles. Conversely, the general right limiting angle will be the minimum of the five right limiting angles. If we create a set of all five left limiting angles and create a set of right limiting angles, we then need to find the maximum of the first set and the minimum of the second set. These two values will be the lower and upper bounds of the allowed range. In other words, the general allowed range will be from the maximum of the five left limiting angles to the minimum of the five right limiting angles.

This technique can easily be generalized beyond simply the angle. The same method of finding the right limits and the left limits can be used for each parameter. For another parameter, one finds the sign of the slope for each winding from $\mathrm{A}_{\mathrm{ij}}{ }^{-1}$ where " $\mathrm{j}$ " indicates the varying parameter. And instead of setting the $i^{\text {th }}$ current equal to \pm 600 amps in equation (15) to find the right and left limits, one uses either (16) or (17) as appropriate.

This gives us a basic scheme for calculating the range: Test the sign of $\mathrm{A}_{\mathrm{ij}}{ }^{-1}$ for each "i," populate a list of left limits and of right limits, and then return the maximum of the left limits and the minimum of the right limits. This routine for a variable angle can be implemented in Mathematica as follows:

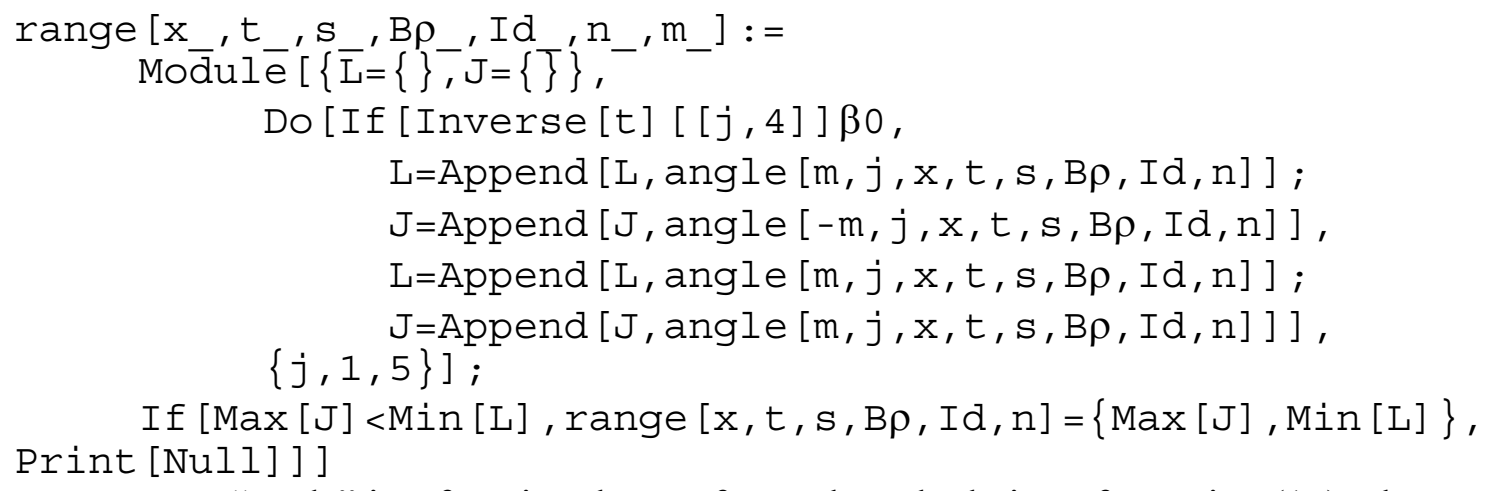

Here "angle" is a function that performs the calculation of equation (15) where " $\mathrm{m}$ " is the max current, " $\mathrm{t}$ " is the transfer matrix and " $\mathrm{n}$ " is the winding factor in equation 
(10). The "Do" loop repeatedly tests the sign of the matrix element $\mathrm{A}_{\mathrm{j} 4}{ }^{-1}$ using an "If" statement. If $\mathrm{A}_{\mathrm{j} 4}{ }^{-1}$ is negative then the value of "angle" at $\mathrm{m}$ amps (in our case 600) is added to the list " $L$ " and the value of "angle" at - $\mathrm{m}$ amps (again -600) is added to the list "J." If $\mathrm{A}_{j 4}{ }^{-1}$ is positive then the value of "angle" at $\mathrm{m}$ amps (600) is added to the list "J" and the value at -m amps (-600) is added to the list "L." Now "J" is all the right limit angles and " $\mathrm{L}$ " the list of the left limit angles. The lower bound on the allowed region will then be $\max [\mathrm{J}]$ and the upper bound will be $\min [\mathrm{L}]$. If $\max [\mathrm{J}]<\min [\mathrm{L}]$, then the allowed region is empty and the above function outputs "Null." If not, then the function outputs the orders pair, $(\max [\mathrm{J}], \min [\mathrm{L}])$, which the allowed range.

While the above function gives the range of allowed values of $x^{\prime}$, it is straightforward to create analogous functions to find the range of $x$ [D3] and $x[D 6]$. The changes that must be made in this case are, that the function "angle" must be changed for the appropriate function, either equations (16) or (17), and the matrix element whose sign is tested must be changed to $\mathrm{A}_{\mathrm{i} 3}{ }^{-1}$ for $\mathrm{x}[\mathrm{D} 3]$ and $\mathrm{A}_{\mathrm{i} 5}{ }^{-1}$ for $\mathrm{x}[\mathrm{D} 6]$. This can all be combined into one function by adding the input value "l" as the position in the displacement vector of the variable parameter. Specifically for $\mathrm{x}[\mathrm{D} 3], 1=3$; for $\mathrm{x}^{\prime} 1=4$; and for $\mathrm{x}[\mathrm{D} 6], 1=5$. The function made must then call a different "Do" loop for each value of "l." In Mathematica this can be implemented like this:

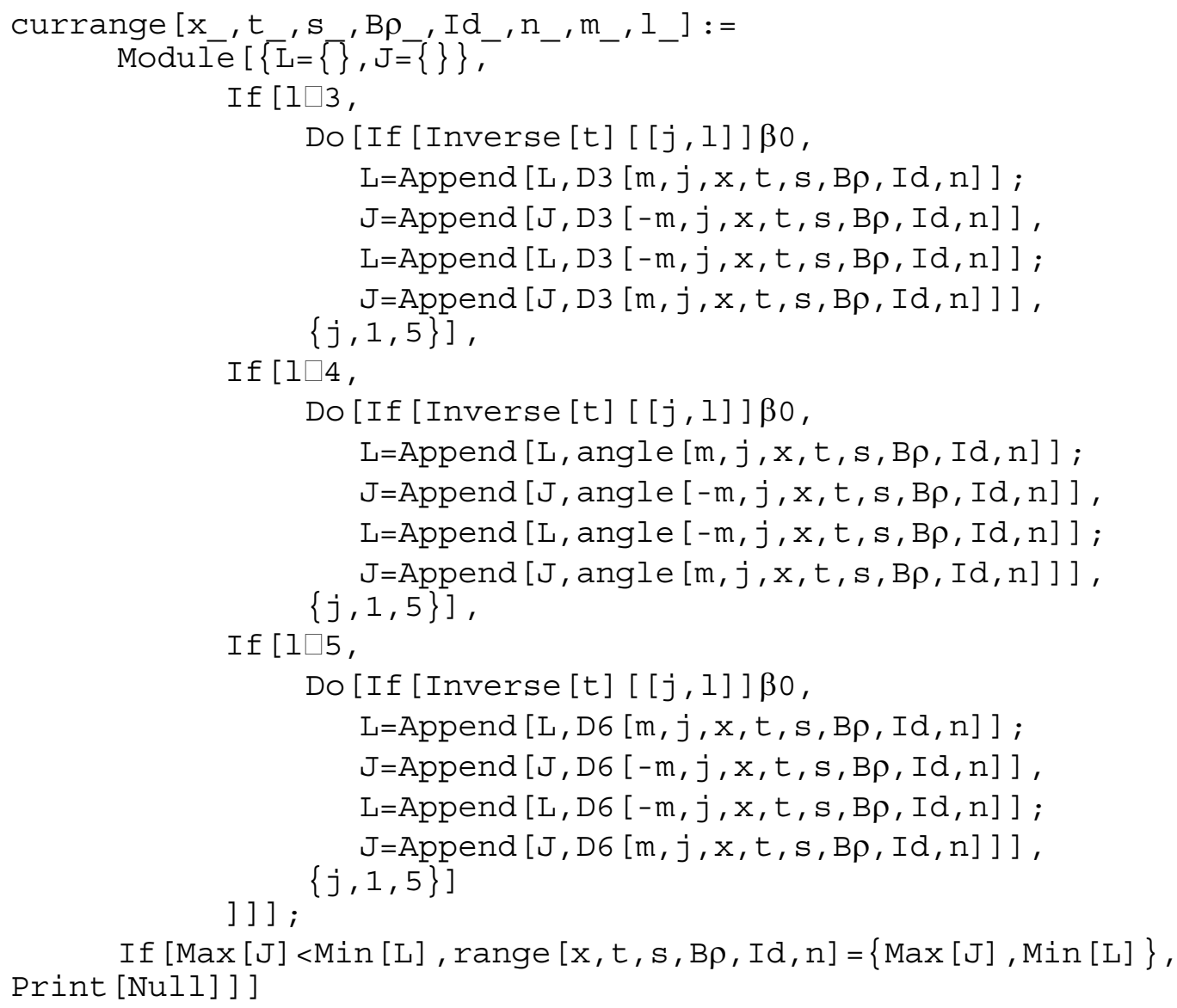

Here, nested "If" statements test the value of "l" and then use the appropriate "Do" loop, which follows an analogous process as used in the "range" function described above. The functions D3, D6 and angle correspond to equations (16), (17) and (15) respectively. 


\section{Exceeding Power Supply Limits}

Knowing this range does not prevent a user from requesting a configuration that exceeds power supply limits. When this happens, it is important to understand the effect on the orbit. The system is designed to set any current that exceeds the power supply limits to the nearest limit instead. For example, a requested 800 amps will become 600 amps, and a requested -700 amps becomes -600 amps. This will clearly lead to two problems: first, the requested offsets are no longer achieved, and second, the bump may no longer be closed.

Understanding the first is fairly simple by inverting the procedure outlined in section II starting with:

$$
\theta_{i}=-\frac{\left(B\left(I_{\text {main }}+I_{i}\right)-B\left(I_{\text {main }}\right)\right) \Delta s}{B \rho}
$$

Here $\theta_{i}$ and $I_{i}$ are the kick and current in the $i^{\text {th }}$ winding respectively. The five kick angles calculated from the currents in (19) can now easily be turned into an offset vector by equation (7).

Returning an estimate for the residuals is, however, not as straightforward. To accomplish this, we first notice that a solution to equation (9) will yield a transverse displacement of zero outside the bump, meaning that equation (5) will be zero for all s outside the bump. This fact allows us to analyze the effects of deviation from the desired field strength in a winding. For example, when a winding is desired to be at a current above 600 amps but is set to 600 amps due to the limit of the power supplies.

If the current at any winding is set to a new current I', this yields a new kick $\theta^{\prime}$. This new kick can be expressed as the old kick plus the change in kick $\delta \theta$ :

$$
\theta_{i}{ }^{\prime}=-\frac{\Delta s \cdot B\left(I_{\text {main }}+\frac{2}{16} I_{i}^{\prime}\right)}{B \rho}=\theta_{i}+\delta \theta_{i}
$$

To calculate the effect of this change in winding current, we can substitute the new kicks $\theta$ ' into the exact solution for the orbit, equation (5):

$x_{c}(s)=\frac{\sqrt{\beta(s)}}{2 \sin (\pi v)} \sum_{i=1}^{n} \sqrt{\beta\left(s_{i}\right)} \theta_{i} \cos \left(\left|\phi(s)-\phi\left(s_{i}\right)\right|-\pi v\right)+\frac{\sqrt{\beta(s)}}{2 \sin (\pi v)} \sum_{i=1}^{n} \sqrt{\beta\left(s_{i}\right)} \delta \theta_{i} \cos \left(\left|\phi(s)-\phi\left(s_{i}\right)\right|-\pi v\right)$

For the desired kicks, the orbit displacement must be zero outside the bump. This means for all $\mathrm{s}$ outside the bump $\mathrm{x}_{\mathrm{c}}(\mathrm{s})$ is reduced to:

$$
x_{c}(s)=\frac{\sqrt{\beta(s)}}{2 \sin (\pi v)} \sum_{i=1}^{n} \sqrt{\beta\left(s_{i}\right)} \delta \theta_{i} \cos \left(\left|\phi(s)-\phi\left(s_{i}\right)\right|-\pi v\right)
$$


However, this exact solution is computationally unwieldy because it requires knowledge of both $\beta(\mathrm{s})$ and $\phi(\mathrm{s})$. If we take the exact solution to the orbit as a psuedoharmonic betatron oscillation, then Sands has an effective remedy to this problem. He states that "for many purposes it is convenient - and sufficient - to approximate betatron motion by a simple harmonic oscillation." ${ }^{5}$ This is done by taking the average $\beta,<\beta>=$ $\beta_{\mathrm{n}}$, over the ring and using $1 / \beta_{\mathrm{n}}$ as the phase advance $\phi$, giving:

$$
x_{c}(s) \approx \frac{\sqrt{\beta_{n}}}{2 \sin (\pi v)} \sum_{i} \sqrt{\beta\left(s_{i}\right)} \delta \theta_{i} \cos \left(\left|\frac{s}{\beta_{n}}-\phi\left(s_{i}\right)\right|-\pi v\right)
$$

This representation will on average stay in phase with the actual orbit. However, since we have taken the amplitude to depend on the average $\beta$, this approximation may yield smaller maxima than the actual orbit. In order to give a high estimate of the amplitude of the residuals, it is more effective to use the maximum of $\beta, \beta_{\max }$, in the amplitude. The approximation now reads:

$$
x_{c}(s) \approx \frac{\sqrt{\beta_{\max }}}{2 \sin (\pi v)} \sum_{i} \sqrt{\beta\left(s_{i}\right)} \delta \theta_{i} \cos \left(\frac{s}{\beta_{n}}-\phi\left(s_{i}\right)-\pi v\right)
$$

Note that the absolute values in the arguments have been removed since outside of the bump, $s / \beta_{n}-\phi_{i}$ will always be positive. In order to find the value of the extrema for this, we can follow the standard maximization procedure and set the derivative equal to zero and substitute the solution for $\mathrm{s}$ into (21). Since we have taken $\beta$ to be constant, the derivative is more straightforward than equation (6):

$$
\begin{aligned}
x_{c}^{\prime}(s)= & -\frac{\sqrt{\beta_{\max }}}{2 \sin (\pi v) \beta_{n}} \sum_{i} \delta \theta_{i} \sqrt{\beta_{i}} \sin \left(\frac{s}{\beta_{n}}-\phi_{i}-\pi v\right) \\
& \Rightarrow 0=\sum_{i} \delta \theta_{i} \sqrt{\beta_{i}} \sin \left(\frac{s_{o}}{\beta_{n}}-\phi_{i}-\pi v\right)
\end{aligned}
$$

where $\mathrm{s}_{\mathrm{o}}$ is the $\mathrm{s}$ coordinate of an extremum. To solve this, we notice that:

$$
\sin \left(\frac{s}{\beta_{n}}-\phi_{i}-\pi v\right)=\sin \left(\frac{s}{\beta_{n}}-\pi v\right) \cos \left(\phi_{i}\right)-\cos \left(\frac{s}{\beta_{n}}-\pi v\right) \sin \left(\phi_{i}\right)
$$

which implies that:

\footnotetext{
${ }^{5}$ M. Sands, The Physics of Electron Storage Rings, SLAC report 121, 1970, p. 41.
} 


$$
\begin{gathered}
0=\sin \left(\frac{s_{o}}{\beta_{n}}-\pi v\right) \sum_{i} \delta \theta_{i} \sqrt{\beta_{i}} \cos \left(\phi_{i}\right)-\cos \left(\frac{s_{o}}{\beta_{n}}-\pi v\right) \sum_{i} \delta \theta_{i} \sqrt{\beta_{i}} \sin \left(\phi_{i}\right) \\
\Rightarrow \tan \left(\frac{s_{o}}{\beta_{n}}-\pi v\right)=\frac{\sum_{i} \delta \theta_{i} \sqrt{\beta_{i}} \sin \left(\phi_{i}\right)}{\sum_{i} \delta \theta_{i} \sqrt{\beta_{i}} \cos \left(\phi_{i}\right)}=L \\
\Rightarrow s_{o}=\beta_{n}[\arctan (L)+\pi v]
\end{gathered}
$$

Substituting back into equation (21), we find that:

$$
x\left(s_{o}\right)=\frac{\sqrt{\beta_{\max }}}{2 \sin (\pi v)} \sum_{i} \delta \theta_{i} \sqrt{\beta_{i}}\left(\cos [\arctan (L)] \cos \left(\phi_{i}\right)+\sin [\arctan (L)] \sin \left(\phi_{i}\right)\right)
$$

and recalling that:

$$
\begin{aligned}
& \cos [\arctan (a / b)]=\frac{b}{\sqrt{a^{2}+b^{2}}} \\
& \sin [\arctan (a / b)]=\frac{a}{\sqrt{a^{2}+b^{2}}}
\end{aligned}
$$

we can see that:

$$
x\left(s_{o}\right)=\frac{\sqrt{\beta_{\max }}}{2 \sin (\pi v)} \sum_{i} \delta \theta_{i} \sqrt{\beta_{i}}\left[\frac{\cos \left(\phi_{i}\right) \sum_{j} \delta \theta_{j} \sqrt{\beta_{j}} \cos \left(\phi_{j}\right)+\sin \left(\phi_{i}\right) \sum_{j} \delta \theta_{j} \sqrt{\beta_{j}} \sin \left(\phi_{j}\right)}{\sqrt{\left(\sum_{i} \delta \theta_{i} \sqrt{\beta_{i}} \sin \left(\phi_{i}\right)\right)^{2}+\left(\sum_{i} \delta \theta_{i} \sqrt{\beta_{i}} \cos \left(\phi_{i}\right)\right)^{2}}}\right]
$$

Since:

$$
\left(\sum_{i} a_{i}\right)^{2}=\sum_{i j} a_{i} a_{j}
$$

where $i$ and $j$ are independent indices, we find that:

$$
x\left(s_{o}\right)=\frac{\sqrt{\beta_{\max }}}{2 \sin (\pi v)}\left[\sum_{i j} \delta \theta_{i} \delta \theta_{j} \sqrt{\beta_{i}} \sqrt{\beta_{j}} \cos \left(\phi_{i}-\phi_{j}\right)\right]^{1 / 2}
$$

This gives us a maximum estimate for the value of the residuals outside of a bump given a deviation from their appropriate current. This equation can be implemented for 
any situation where the actual current in the winding differs form the desired current. This becomes particularly useful when the desired current exceeds the physical limit of the power supplies, giving us an estimate for the residuals outside the bump. With equation (24) along with equations (19) and (7) we can describe the effects of requesting unallowed values of the power supplies.

The accuracy of these two techniques can be tested using MAD by evaluating the transverse orbit once power supplies have been requested to exceed their limit. In order to achieve $3 \mathrm{~mm}$ offsets at D3 and D6, and an angle of $0 \mathrm{mrad}$ at D6 with a dipole current of $4723 \mathrm{amp}$, one must set the C7 power supply to $-645.21 \mathrm{amps}$, and the D4 power supply to -645.953 amps. If these bump parameters are requested these two power supplies will be set to -600 amps instead. Equations (19) and (7) predict that this will give an offset of $2.1 \mathrm{~mm}$ at D3 and $2.7 \mathrm{~mm}$ at D6, and an angle of $0.072 \mathrm{mrad}$ at D6. Equation (24) predicts that these currents will yield residuals outside the bump of 5.24 $\mathrm{mm}$. We can see below the orbit as simulated by MAD using the same settings used in Figure 2:

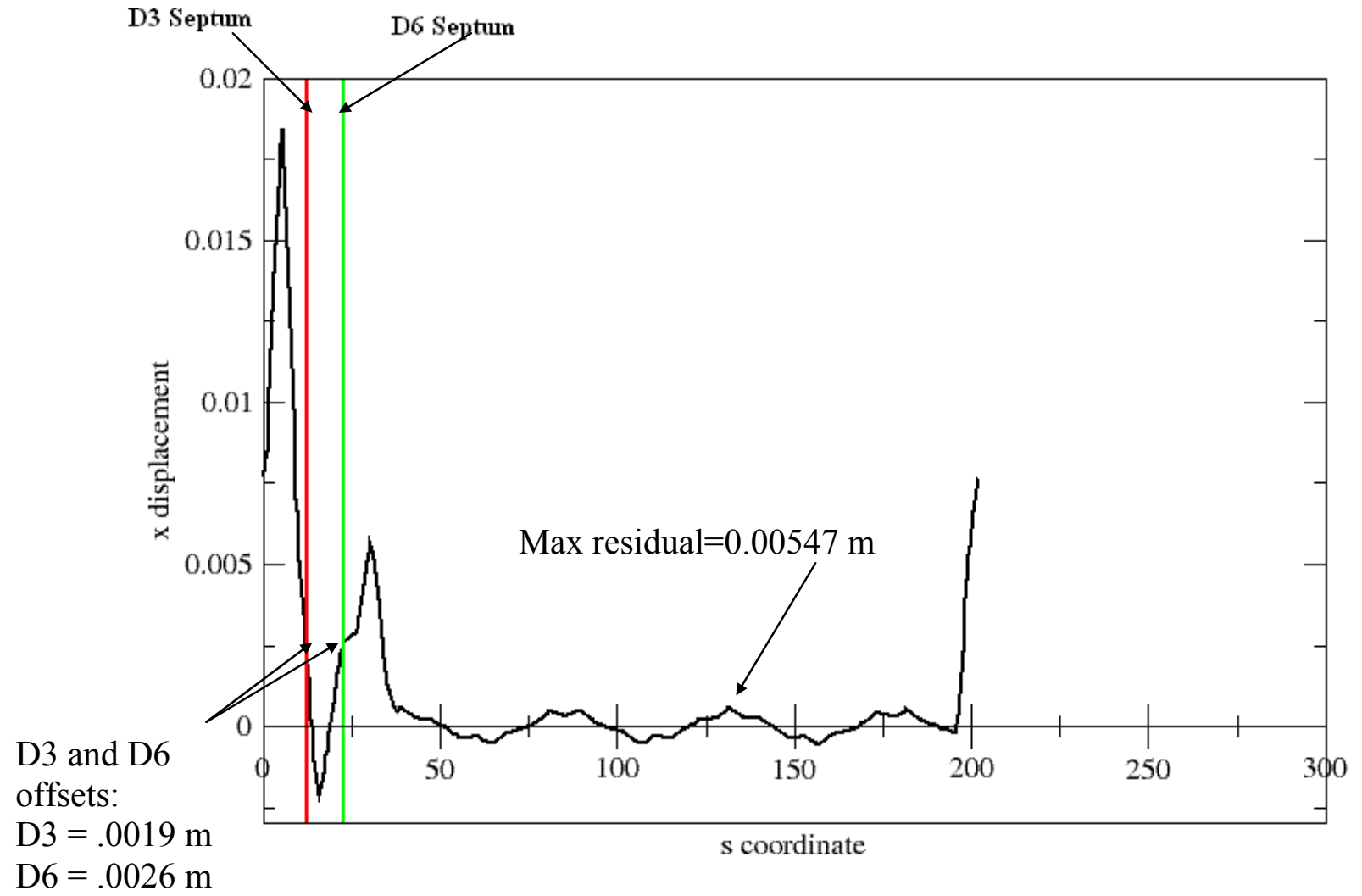

Figure 8. Horizontal transverse orbit generated by MAD.

Main dipole current at 4723 amps and offsets set to $3 \mathrm{~mm}$ at each septum and the angle at D6 set to $0 \mathrm{mrad}$. These parameters exceed power supply limits.

We can see that the actual calculated results are close to the prediction. The offset at D3 was calculated to be $1.9 \mathrm{~mm}$ and the offset at D6 was calculated to be $2.6 \mathrm{~mm}$. While not shown in the figure, the angle at D6 was calculated to be $0.087 \mathrm{mrad}$. The calculated value of the residual, $5.47 \mathrm{~mm}$, also agrees well with the prediction. 


\section{Further Development}

While the techniques developed above are sufficient for the full functionality of the new interface, there are four possible areas of improvement that they expose. The first is a way to allow a user to specify their bump parameters and allow the bump not to be closed and solve for a set of winding currents which minimizes residuals but does not exceed power supply limits. The second is the possibility of integrating the beam position monitoring system into the interface. The third is a way to deal with distortions in the closed orbit. The fourth is a method of calculating the ideal angle at D3 given the set skew of the D3 septum. This may also include a calculation of the effects on extraction efficiency due to changes in the angle at D3. These possibilities are outlined and some guidance is given below.

Since a large range of the possible bump configurations are restricted due to the power supply limit it may be advantageous for a user to eliminate the condition that the bump be closed, in favor of achieving specific offsets. In this case, it will be desirable to achieve the bump parameters set, remain within the power supply limits and minimize the residuals. Together, these three tasks should be sufficient to give a unique set of currents given two offsets.

Removing the boundary conditions, that the position and angle must be zero at $\mathrm{E} 1$, is equivalent to removing the first two rows from the matrix $\mathrm{A}$ in equation (7). The system can now be described as an underdetermined system of linear equations following this matrix equation with $\mathrm{B}$ as a $3 \times 5$ matrix equivalent to the bottom three rows of $\mathrm{A}$ :

$$
\left(\begin{array}{c}
x_{D 3} \\
x_{D 6}^{\prime} \\
x_{D 6}
\end{array}\right)=\left(\begin{array}{l}
\quad B \\
\theta_{5}
\end{array}\right)
$$

In general, there will be infinitely many solutions for the vector $\theta$. However, with the two conditions mentioned above, it may be possible to find a unique solution. The condition of the power supply limit can be written as:

$$
\underset{i=1, \ldots, 5}{\operatorname{aax}}\left\{\left|I_{i}\right|\right\} \leq 600
$$

To write the second condition, minimum residuals, we can find an expression for the amplitude of the residuals given the kick angles in the kick vector from equation (24). Here, we will use the same form of approximation except, instead of a change in angle $\delta \theta$, we will simply use the kick angle $\theta$ :

$$
x\left(s_{o}\right)=\frac{\sqrt{\beta_{\max }}}{2 \sin (\pi v)}\left[\sum_{i j} \theta_{i} \theta_{j} \sqrt{\beta_{i}} \sqrt{\beta_{j}} \cos \left(\phi_{i}-\phi_{j}\right)\right]^{1 / 2}
$$


The goal now becomes the determination of the kick vector which satisfies equations (25) and (26) as well as minimizing (27). It should also be noted that the space of kick vectors is not properly five-dimensional. Since the kick vector must satisfy (24), provided that the matrix B is of full rank, one need only determine the values of three kick vectors to find the value of all five. Therefore, the job of minimizing (27) is over this three-dimensional space, something which maybe possible using known optimization techniques. The constraint from (26) is crucial but this simply gives bounds to the space over which (27) must be optimized and so does not require the development or employment of new techniques. Once an appropriate optimization technique is found this problem will be resolved, and it will be possible to determine the kick vector which will both satisfy (25) and (27).

Secondly, as noted above, it may be helpful for a user to receive feedback as to what the BPM system says in comparison to the calculations. Since the BPM system is known to contain error, this comparison should not necessarily be biased either way, rather it should present the user with the BPM data that corresponds to the calculations. There are two possibilities for this. One simple way would be to present the BPM data for the key calculated values as a list, the offsets at the septa, the angle at one septum, the kicks caused by the trim windings, and the amplitude of the residuals. Another possibility would be to present parallel graphs from the calculation and the BPM system, one of the transverse orbit according to the BPM system and another of the calculated orbit according to equation (5), which requires values of $\beta$ and $\phi$ at several s coordinates.

The third possible area of improvement centers on the fact that equations (5) and (6) are measured with respect to a reference orbit. In the case of the extraction in the Booster, this reference orbit is the orbit prior to turning on the trim windings of the bump. Ultimately we are concerned with the distance from D3 and D6 septa as well as the absolute angle at each position. However, we have calculated the angle and offset in relation to the reference orbit. If the reference orbit is flat and centered in the beam pipe then these two are equivalent, but this may not be the case. While trim windings correct the orbit at injection, orbit disturbances scale with energy, and so the orbit may not be flat at extraction. It would be helpful to be able to integrate the nature of these distortions into the user interface for the extraction bump.

Fourth, the central role of the bump is to control extraction by controlling the position and angle of the equilibrium orbit relative to the septa. The angle of the orbit proves particularly problematic because it characterizes how the position relative to the septum changes from the upstream to downstream ends of the septum. For simplicity, the angle chosen should keep this relation constant. There are three main factors that affect this relationship: first, the angle of the reference orbit; second, the angle of the equilibrium orbit defined by the bump; and third, the phase advance across the septum. The first two combine to change the transverse position in the beam pipe across the septum and the third position of the upstream and downstream end of the septum relative to the phase space diagram of the beam. Since this angle changes with energy and the configuration of the accelerator, it would be nice to have a way to automatically calculate this angle and preset it into the orbit control program.

The first and third suggestions are most pertinent to study out of the four presented. The distortions in the reference orbit taken into account in the third suggestion change the effectiveness of the bump. With knowledge of these distortions, the user will be able to more effectively integrate the bump into an overall extraction scheme. The 
alteration of boundary conditions outlined in the first suggestions could add a large range of possible bump configurations depending on how small the residuals can be made.

This would do more than increase the functionality of the interface program; by increasing the number of practical accelerator settings it increases the range of possible operating parameters for the accelerator, without any hardware improvements. Also, the techniques developed may be relevant to similar problems throughout the accelerator and would be a vital addition to techniques at hand for developing control systems.

\section{Implementation}

Using the techniques developed above this section will outline a possible new user interface for the control of the NSRL extraction bump. First, the appearance and functionality of the interface will be explained, next the flow of inputs and outputs through the program will be outlined, then the equations used will be presented, and finally the procedure for executing the calculations will be detailed.

\section{User Interface}

Here is an image of the user interface.

\section{First Window:}

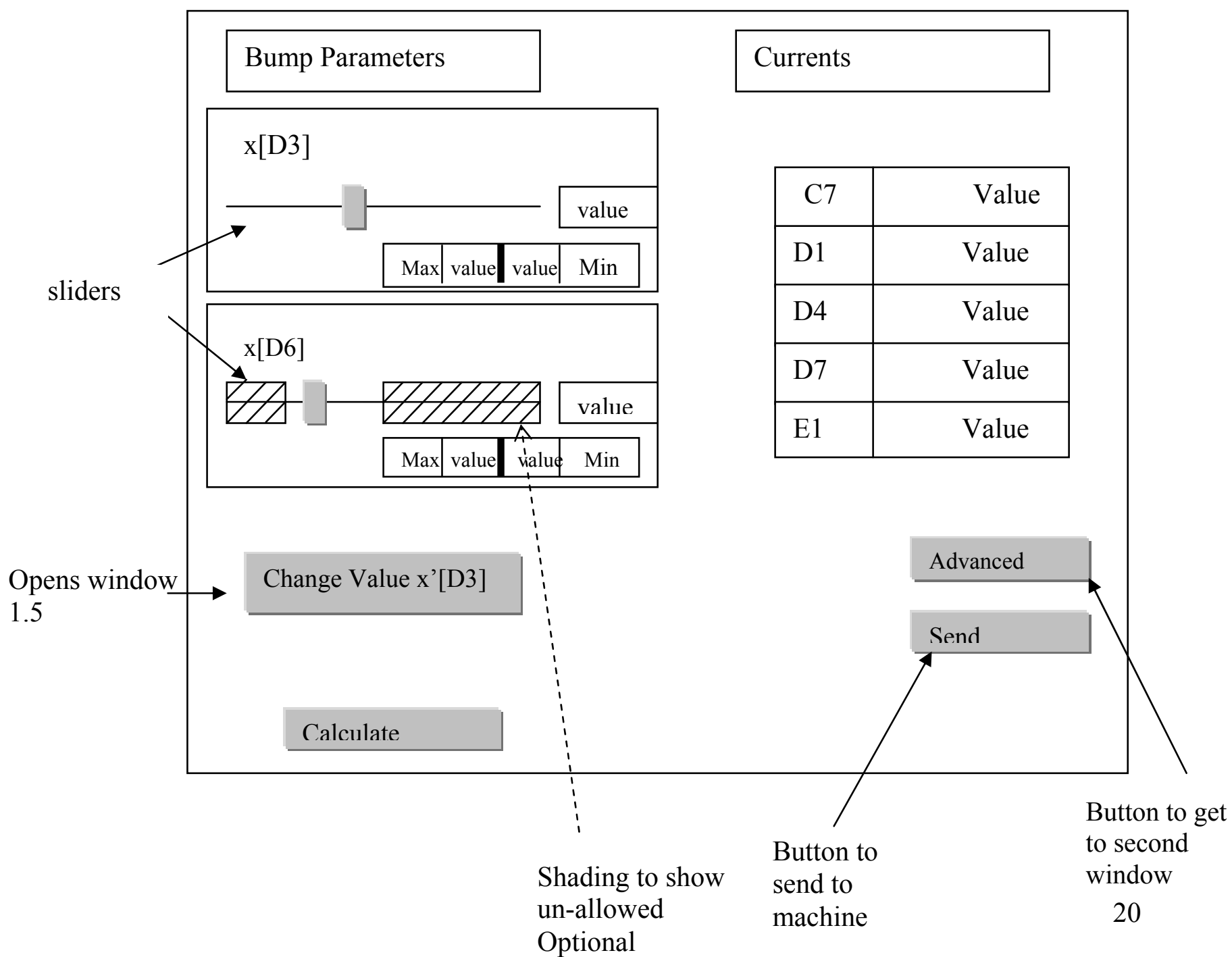




\section{Changing Angle at D3: Window 1.5}

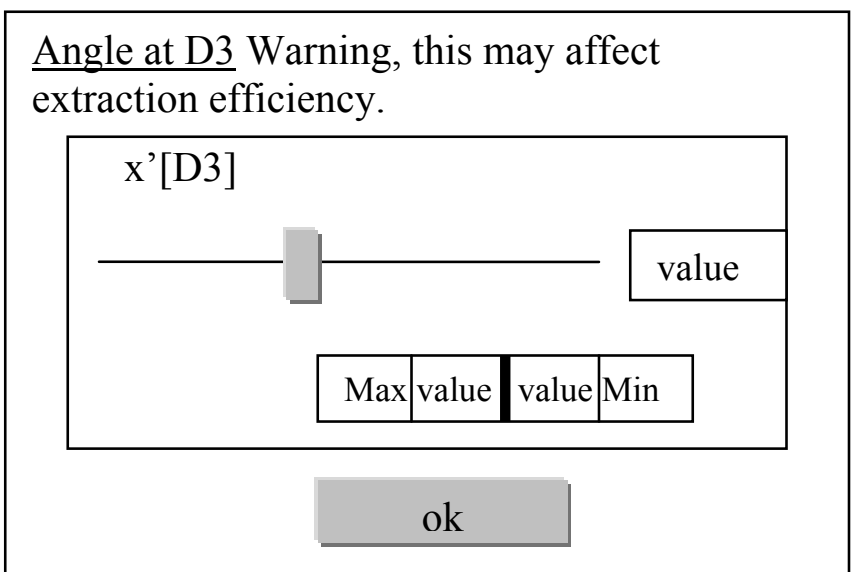

Second Window:

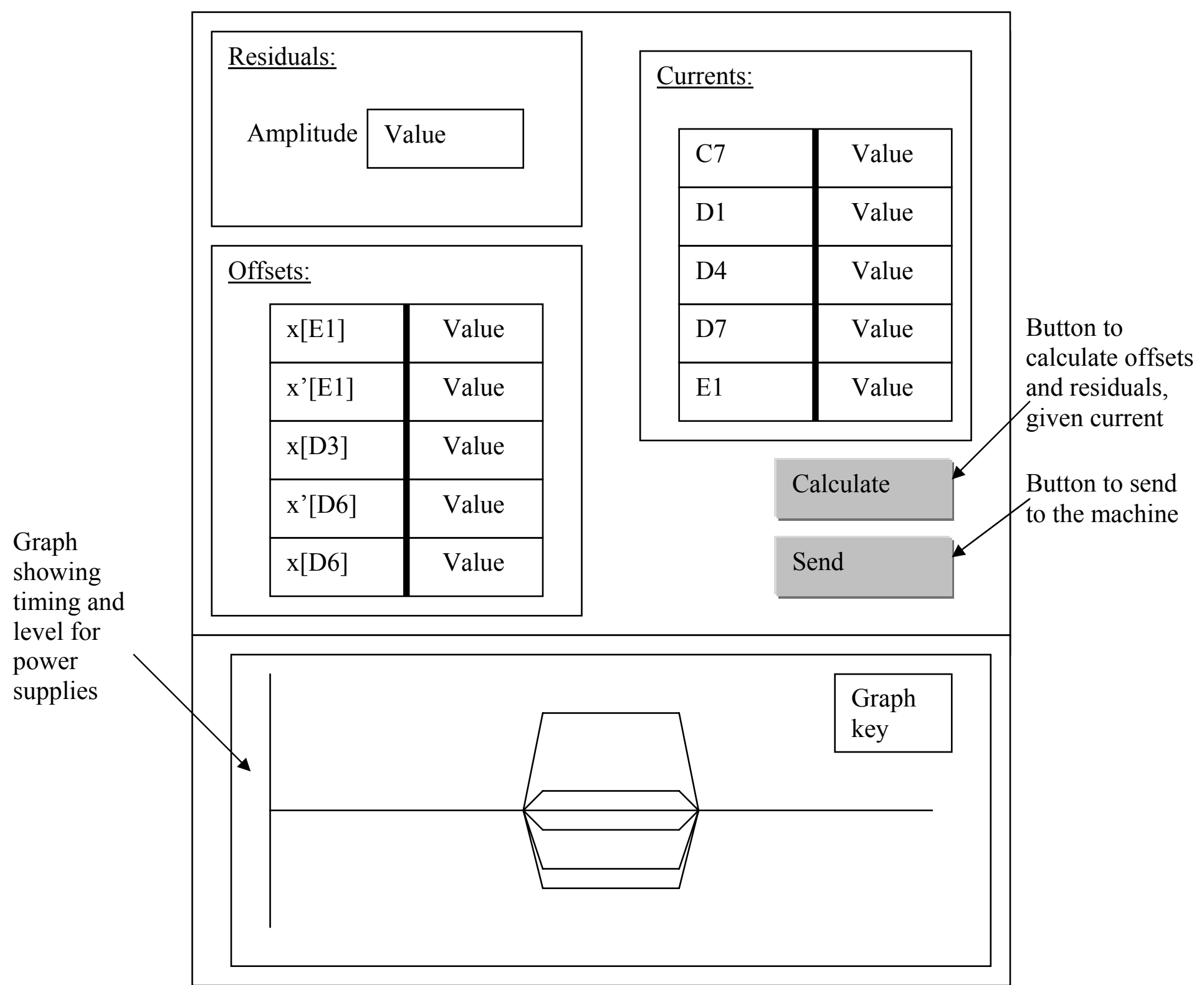


The most common usage of this interface will be to set offsets for the bump. The design shown above accomplishes this as well as several extra functions. The first window allows the user to input the offsets and calculate the necessary currents. The angle at D3 will be preset and can be changed in window 1.5, which is accessed through a button on the first window. Once one offset is set, the allowed range (given the hidden setting of the angle and determined by the physical limits of the power supplies) of the other can be calculated. So long as the currents are allowed, they can be sent to the power supplies from this window. This level of functionality should be sufficient for most users. However, the above design offers more options through the second window. In the second window, the user is presented with the consequences of power supplies being requested to exceed their limit. This window also allows the user to change the currents directly and observe how different current combinations will affect the bump parameters and what the orbit outside of the bump will look like. Below, the composition and use of each window will be explained in more detail.

\section{First Window:}

This window allows a user to input offsets and calculate either a range for one offset or the currents needed to achieve the requested bump. The offsets will be input using two methods. For quick visual reference sliders can be used. Each slider will have a range centered at zero that encompasses the most common setpoints for each parameter. The range of the $x[D 3]$ slider and the $x[D 6]$ slider will be from $-2 \mathrm{~cm}$ to $+2 \mathrm{~cm}$. There is also a field that numerically gives the value of the offset. Ideally, this field would be dynamically refreshed as the corresponding slider is moved. This field should also act as an input for numerical values of the offset. The "clear" button shown will remove any value from that offset. This allows a user to set the value for one offset, then clear the other, enabling them to find the allowed range of the second offset using the calculate button.

From the first window, the user can calculate the required current for a given configuration but they can also calculate the allowed range of one offset given the value of one. Both of these functions should be controlled using the "calculate" button. The normal order of use for this window will be that the user specifies an offset, presses the "calculate" button, and is shown the allowed range for the third. Then the user will set the third parameter within the allowed range, again press "calculate" and be returned the list of necessary currents. They will then press the "send" button once everything is to their liking and the currents will be sent to the power supplies. The allowed ranges will be output in different ways, graphically, using shaded regions on the slider bars, and numerically, using fields below the sliders. The currents will be shown in the fields to the right.

The allowed range is calculated using two pieces of information, the power supply limit and the preset value of the angle at D3. When the user presses calculate, the program will take the offset given, the preset angle, and the $\pm 600 \mathrm{amp}$ limit of the power supplies and find the range of the second offset that will achieve the preset angle at D3.

If a user sets the second offset outside the allowed range, then, once they press calculate, they will be prompted with two dialog boxes: one explaining they cannot achieve the preset angle and how they can change that preset, and another telling them which power supplies are set to exceed their limit. The second dialog box will give the requested value and explain that this value will be changed to the nearest limit. An example of the two boxes can be seen below: 
Warning

Window 1

Warning

Window 2
Warning: The preset angle at D3 cannot be achieved with the offset chosen due to power supply limits. If you wish to keep this offset, your range of allowed angles will be restricted. To change the preset angle press the "Change Value $\mathrm{x}$ '[D3] button.

\section{ok}

Warning: you have requested currents that exceed power supply limits. If you wish to assign these currents, please use the advanced option.

IC7 $=800 \mathrm{amps}$

Will be set to $600 \mathrm{amps}$

$$
\text { ID4=-750 amps }
$$

Will be set to -600 amps

\section{ok}

Once the user closes both windows they will see the requested list of currents output on the right-hand side of the window, including those currents that exceed the power supply limit. The problematic currents should be flagged by changing the background color of the field they are in. From the first window, a user cannot send an unallowed set of currents to the power supplies. If they have requested an unallowed set of currents, they must open the second window in order to send them to the power supplies.

After the user closes the warnings, they may want to change the angle at D3 and see what range their chosen offset allows for $\mathrm{x}$ '[D3]. To do this they press the button marked "Change Value $\mathrm{x}$ '[D3]" below the offset sliders in the first window. This will open window 1.5. In window 1.5 there will be an equivalent interface for the offsets, there will be a slider, a field with the numerical value, and fields for the maximum and the minimum of allowed values. The slider will have a range of $\pm 2.5 \mathrm{mrad}$, and the numerical field will function the same as for the offsets; it will both provide for input and dynamic output. If the user has opened this window without setting the two offsets, the allowed range fields will be empty; however, if the user has already set the two offsets, then the allowed range (according to power supply limits) will be given. The "ok" button 
in window 1.5 performs one of three operations: If no other offset has been set, then it simply closes the window and sets the angle to whatever the user specified. If one other offset has been set, then it calculates the allowed range for the third and if both other offsets have already been set, then it calculates the required currents. If the both offsets have been set and the user specifies a value for the angle that exceeds power supply limits, then the user will be prompted with the second warning window.

\section{Second Window}

The second window is accessed through a button in the first window labeled "advanced" and offers more options to the user. Here, the user is presented with information characterizing the consequences of exceeding power supply limits and has the ability to directly input currents and see the reduced list of currents. A "clear" button serves to clear the values for all currents at once. There are two scenarios in which a user will use this screen: first, when they have requested an unallowed set of currents and would like to see what the consequences are; and second, when they would like to input the currents directly and observe the effect on the bump parameters and the residuals.

The first scenario will occur when a user has requested a configuration of offsets and angles using the first window and been prompted with the warning dialog box. They will then press the "advanced" button and proceed to the second window. Once the user presses the "advanced" button, the currents calculated from the first window will be used to perform the calculations necessary for the outputs in the second window. This means that when the second window is displayed it will display the results associated with the inputs in the first window. In the second window, the user will see the altered list of currents that would be sent to the power supplies (where all values that exceed the limit have been changed to the nearest limit). They will also see the values of the bump parameters that this new list of currents will produce as well as the amplitude of the residual displacement of the orbit outside of the bump. At this point, the user can either send this changed set of currents to the power supplies, or they can alter them. The fields that serve as output for the currents will also take inputs of new currents. Once the user has altered the currents, he can press the "calculate" button in the second window to see what the effect on the bump parameters and residuals will be. If the user has again requested unallowed currents they will be prompted by the second warning window. When they close this window the currents shown in the fields will be reduced to allowed values.

The second scenario can occur without any inputs being given in the first window. The user can simply press the "advanced" button and open the second window. From here they can directly input new currents and calculate the offsets and the residual. The functionality at this point will be equivalent to changing currents in the first scenario.

At the bottom of this window there is also a graph displaying the amperage of a power supply with respect to time. This is the same graph currently being used in the NSRL extraction bump control interface and so the insertion of it should follow the same method.

\section{Inputs and outputs:}

This chart attempts to make clear the flow of inputs and outputs. The details as to what inputs feed into what calculation and the precise connection between the calculations will be explained later. 
Input

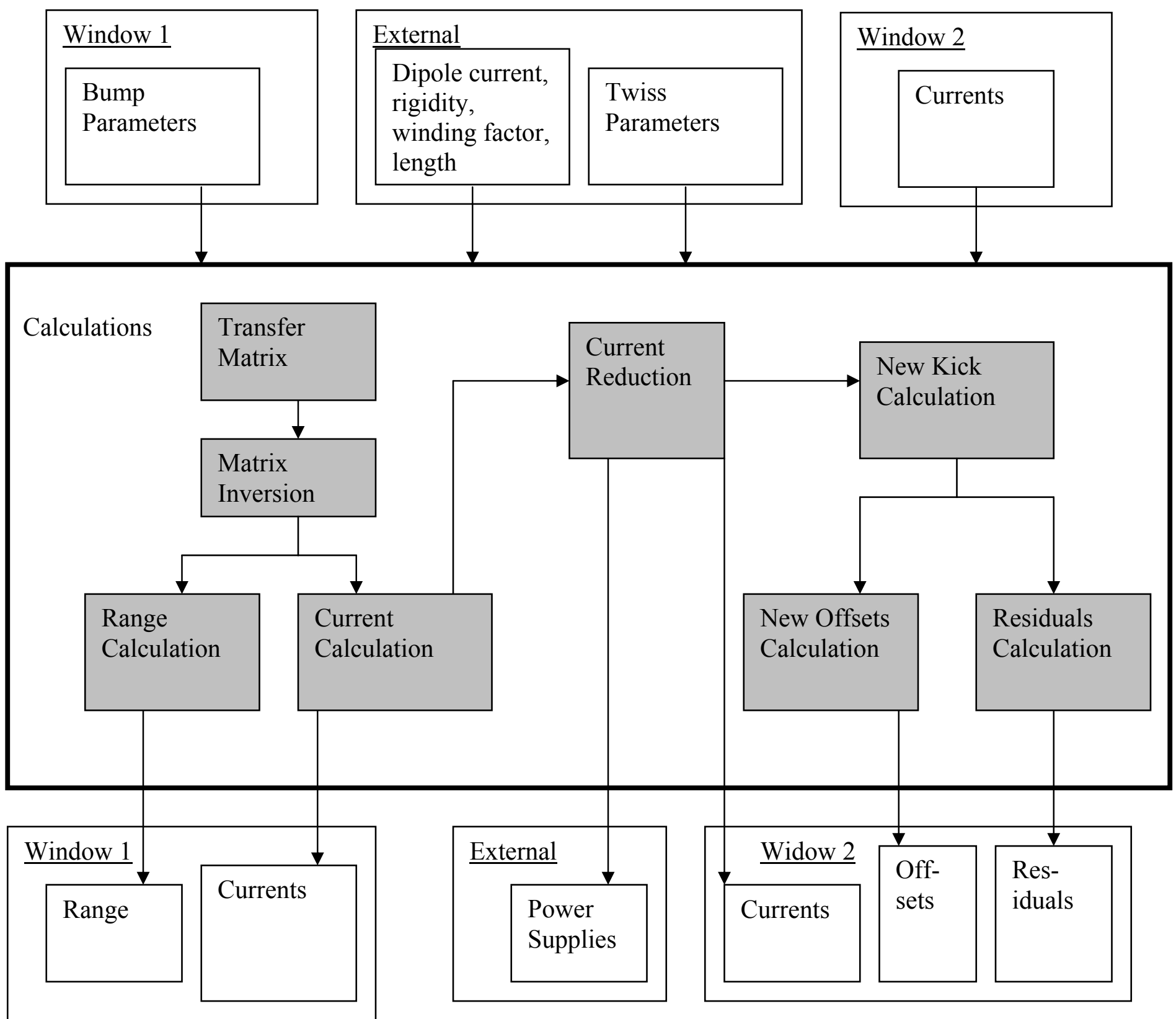

Output

This shows how the outputs for the two windows and the power supplies will be calculated. The first window will input the offsets with the angle preset. The twiss parameters will be taken from an external database and be used to evaluate the transfer matrix, which will then be inverted. This inverse matrix will then be used, along with bump parameters from the first window and the dipole current, rigidity, winding factor, and magnet length (what will here be called the "machine information") from an external 
database, to find the currents. Once one offset is input it will also be used to find the range of the second, or the range of the angle if both offsets are given.

Once the currents are calculated from the transfer matrix they are "reduced" meaning all values that are above the power supply limit are changed to \pm 600 amps. Before any currents are sent to the machine they are reduced to ensure that only allowed values are requested of the power supplies. The calculation that reduces the power supplies will also produce the second warning window discussed above. This reduced list will be used to calculate the new kicks that the windings will cause. In turn, the new kicks will be used to calculate the new offsets and the amplitude of the residuals, shown in the second window.

The details regarding the precise inputs and outputs for each calculation will be discussed below when each calculation is described.

\section{Defining Variables}

For expedient reference, here are the conventions of variable definition that are used in the calculations below (Many have previously been defined in the body of the paper.):

Index Convention: The transfer matrix has been defined such that there is a specific order to the windings that corresponds to the index of the kick and current vectors. This convention is: $1=\mathrm{C} 7,2=\mathrm{D} 1,3=\mathrm{D} 4,4=\mathrm{D} 7,5=\mathrm{E} 1$.

$\mathrm{A}_{\mathrm{ij}}=$ The element of the transfer matrix in the $\mathrm{i}^{\text {th }}$ row and $\mathrm{j}^{\text {th }}$ column.

$\mathrm{A}_{\mathrm{ij}}{ }^{-1}=$ The element of the inverse of the transfer matrix in the $\mathrm{i}^{\text {th }}$ row and the $\mathrm{j}^{\text {th }}$ column.

$\mathrm{x}[\mathrm{D} 3]=\mathrm{x}_{\mathrm{D} 3}=$ The value of the transverse displacement in the $\mathrm{x}$ direction at the septum D3

$\mathrm{x}^{\prime}[\mathrm{D} 3]=\mathrm{x}_{\mathrm{D} 3}=$ The value of the angle of the transverse trajectory in the $\mathrm{x}$ direction at the septum D3

$\mathrm{x}[\mathrm{D} 6]=\mathrm{x}_{\mathrm{D} 6}=$ The value of the transverse displacement in the $\mathrm{x}$ direction at the septum D6

$v=$ The tune

$\beta_{i}=$ The value of the beta-function at the $i^{\text {th }}$ winding

$\beta_{\mathrm{m}}=$ The maximum $\beta$ over the whole machine

$\beta(x)=$ The value of the beta-function at position $x$

$\varphi_{\mathrm{i}}=$ The value of the phase advance at the $\mathrm{i}^{\text {th }}$ winding

$\varphi(\mathrm{x})=$ The phase advance at position $\mathrm{x}$ 
$\theta_{\mathrm{i}}=$ The kick due to the $\mathrm{i}^{\text {th }}$ winding

$I_{i}=$ The current in the $i^{\text {th }}$ winding

$\mathrm{I}_{\mathrm{i}}{ }^{\prime}=$ The reduced current in the $\mathrm{i}^{\text {th }}$ winding

$\mathrm{B} \rho=$ The magnetic ridigity

$I_{\text {main }}=I_{d}=$ The main dipole current

$\mathrm{B}=$ The magnetic field

$\Delta \mathrm{B}_{\mathrm{i}}=$ The change in magnetic field at the $\mathrm{i}^{\text {th }}$ winding

$\mathrm{S}=\Delta \mathrm{s}=$ The length of the magnets

$\mathrm{n}=$ The winding factor, always equals $2 / 16$

\section{Magnetic Field Functions}

Recall the two equation for B(i) and $\mathrm{B}^{-1}$ (i) developed above. These should be stored in the program's memory:

$$
\begin{aligned}
\mathrm{B}(\mathrm{i})= & 0.0009122+0.0002371 \mathrm{i}+1.717 \times 10^{-8} \mathrm{i}^{2}-2.412 \times 10^{-11} \mathrm{i}^{3}+1.836 \times 10^{-14} \mathrm{i}^{4} \\
& -7.88 \times 10^{-18} \mathrm{i}^{5}+1.891 \times 10^{-21} \mathrm{i}^{6}-2.351 \times 10^{-25} \mathrm{i}^{7}+1.163 \times 10^{-29} \mathrm{i}^{8}
\end{aligned}
$$

and

$$
\begin{aligned}
\mathrm{B}^{-1}(\mathrm{x})= & -3.74879+4190.08 \mathrm{x}-643.808 \mathrm{x}^{2}+2578.06 \mathrm{x}^{3}-5126.86 \mathrm{x}^{4} \\
& +4101.54 \mathrm{x}^{5}+1312.0 \mathrm{x}^{6}-3992.35 \mathrm{x}^{7}+1727.21 \mathrm{x}^{8}
\end{aligned}
$$




\section{Equations}

Here is the flow of the equations that will be used. It is recapitulation of what has already been developed and may serve as a useful reference.

\section{Calculating the Currents}

$$
\begin{aligned}
& \left(\begin{array}{c}
0 \\
0 \\
x_{D 3} \\
x_{D 3}^{\prime} \\
x_{D 6}
\end{array}\right)=\left(\begin{array}{lll} 
& \\
& A
\end{array}\right)\left(\begin{array}{c}
\theta_{1} \\
\vdots \\
\theta_{5}
\end{array}\right) \\
& \Rightarrow\left(\begin{array}{c}
\theta_{1} \\
\theta_{2} \\
\theta_{3} \\
\theta_{4} \\
\theta_{5}
\end{array}\right)=\left(\begin{array}{l}
A^{-1} \\
\end{array}\right)\left(\begin{array}{c}
0 \\
0 \\
x_{D 3} \\
x^{\prime} \\
x_{D 6}
\end{array}\right) \\
& \theta_{i}=-\frac{\Delta s \Delta B_{i}}{B \rho} \\
& \Rightarrow \Delta B_{i}=-\frac{\theta_{i} B \rho}{s} \\
& \Delta B_{i}=B\left(I_{d}+n I_{i}\right)-B\left(I_{d}\right) \\
& \frac{1}{n}\left[B^{-1}\left(\Delta B_{i}+B\left(I_{d}\right)\right)-I_{d}\right]=I_{i} \\
& \Rightarrow I_{i}=\frac{1}{n}\left[B^{-1}\left(-\frac{\theta_{i} B \rho}{s}+B\left(I_{d}\right)\right)-I_{d}\right]
\end{aligned}
$$




\section{Calculating the Range}

$$
\begin{gathered}
x^{\prime}=-\frac{1}{A_{i 4}^{-1}}\left(\frac{\Delta s\left(B\left(I_{d}+n I_{i}\right)-B\left(I_{d}\right)\right)}{B \rho}+A_{i 3}^{-1} x_{D 3}+A_{i 5}^{-1} x_{D 6}\right) \\
x_{D 3}=-\frac{1}{A_{i 3}^{-1}}\left(\frac{\Delta s\left(B\left(I_{d}+n I_{i}\right)-B\left(I_{d}\right)\right)}{B \rho}+A_{i 4}^{-1} x^{\prime}+A_{i 5}^{-1} x_{D 6}\right) \\
x_{D 6}=-\frac{1}{A_{i 5}^{-1}}\left(\frac{\Delta s\left(B\left(I_{d}+n I_{i}\right)-B\left(I_{d}\right)\right)}{B \rho}+A_{i 4}^{-1} x^{\prime}+A_{i 3}^{-1} x_{D 3}\right) \\
I_{i}=B^{-1}\left[B\left(I_{\text {main }}\right)-\frac{B \rho}{\Delta s}\left(A_{i 3}^{-1} x_{D 3}+A_{i 4}^{-1} x^{\prime}+A_{i 5}^{-1} x_{D 6}\right)\right]-I_{\text {main }}
\end{gathered}
$$

These are used to calculate the range of allowed currents when two parameters are given.

\section{$\underline{\text { Calculating new kicks }}$}

$$
\begin{gathered}
\Delta B_{i}^{\prime}=B\left(I_{d}+n I_{i}^{\prime}\right)-B\left(I_{d}\right) \\
\theta_{i}{ }^{\prime}=-\frac{\Delta B_{i}{ }^{\prime} s}{B \rho} \\
\theta_{i}{ }^{\prime}=-\frac{\left(B\left(I_{d}+n I_{i}^{\prime}\right)-B\left(I_{d}\right)\right) s}{B \rho}
\end{gathered}
$$

These are the new kicks.

$\underline{\text { New Offsets }}$

$$
\left(\begin{array}{c}
x_{E 1} \\
x_{E 1}^{\prime} \\
x_{D 3} \\
x_{D 3}^{\prime} \\
x_{D 6}
\end{array}\right)=\left(\begin{array}{l}
\theta_{1}^{\prime} \\
\\
\vdots \\
\theta_{5}{ }^{\prime}
\end{array}\right)
$$

This gives the new offsets from the new kicks. 
Amplitude of Residuals

$$
x\left(s_{o}\right)=\frac{\sqrt{\beta_{\max }}}{2 \sin (\pi v)}\left[\sum_{i j} \theta_{i} \theta_{j} \sqrt{\beta_{i}} \sqrt{\beta_{j}} \cos \left(\varphi_{i}-\varphi_{j}\right)\right]^{1 / 2}
$$

This takes the twiss parameters at each winding, the new kicks, and the maximum of the $\beta$-function over the whole ring to give the amplitude of the residuals.

\section{Detail of Calculations}

Following is an explanation for the procedure to execute each calculation described in the input/output figure above. Along with each calculation is a more detailed diagram of the inputs and outputs for that calculation. These diagrams will be of the following form:

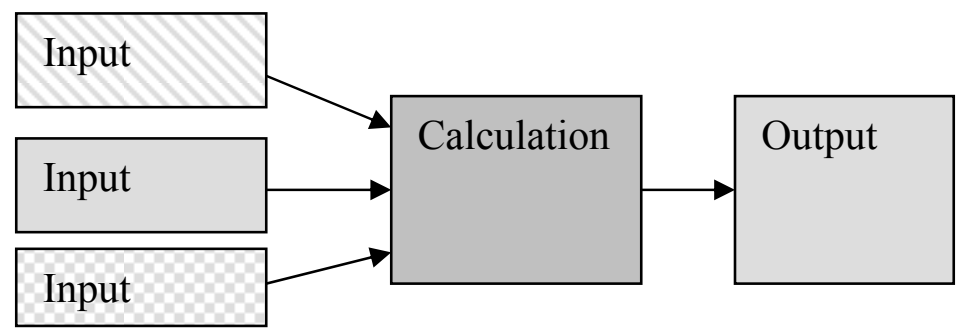

If the input is taken from outside the program, either from a user input or an external database, it is light gray with stripes. If it comes from a calculation internal to the program it is solid light gray, and if it is something that is in the internal memory of the program, then it is light gray checkerboard pattern. The outputs will be solid light gray to indicate that they are calculations internal to the program and can be used as inputs as well.

\section{The Transfer Matrix}

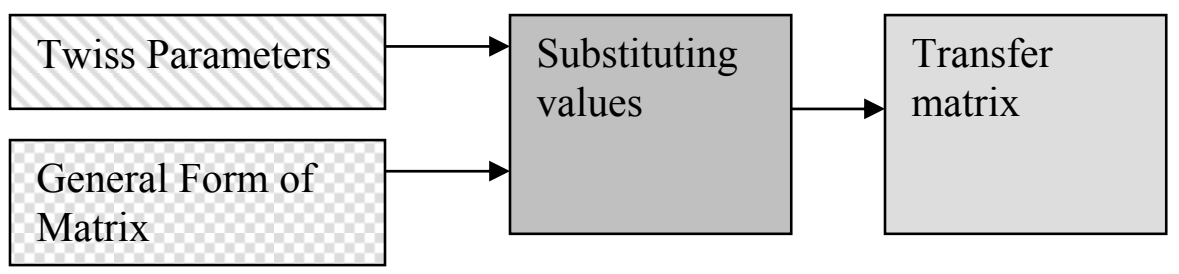

The general form of the transfer matrix can be found in the appendix of this document. The values for the twiss parameters, $\beta, \alpha$, and $\varphi$, will be taken from an external library. These will then be substituted into the general form of the matrix to find the transfer matrix that will be used for all future calculations. 
$\underline{\text { Inverting Matrix }}$

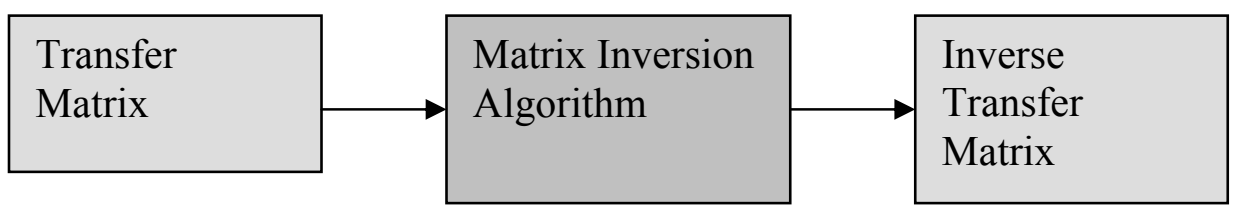

This calculation will use standard matrix inversion algorithms to invert the transfer matrix calculated above.

\section{Calculating the Currents}

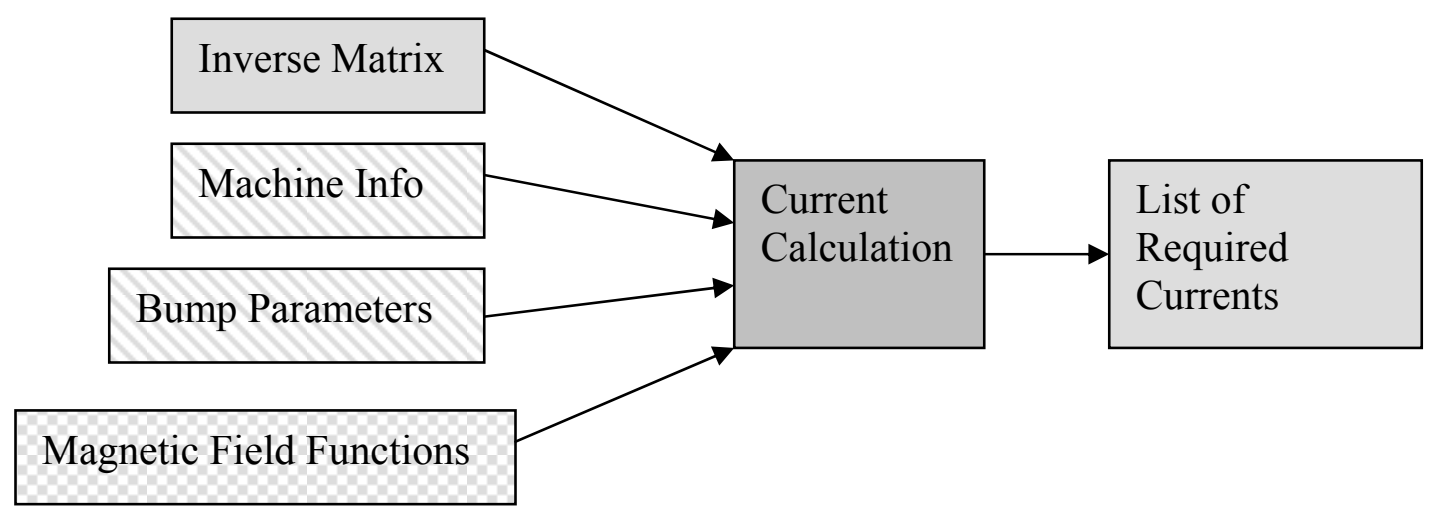

The current calculation will take the inverse matrix calculated above, the machine information, the bump parameters set by the user, and the magnetic field functions (28) and (29) and output the list of currents required to achieve the set bump parameters. Ultimately, this list will be displayed in the first window. Before this however, it will pass through the current reduction calculation (detailed below) to test for requests that exceed the power supply limits. While the current reduction calculation will produce a new list, this new list will only be displayed to the user in the second window.

To find the current from the bump parameters given, you must apply equation (31), with the inverse matrix found above and find the kick angles, then go directly from the kick angles to the currents using equation (36).

\section{The Allowed Range}

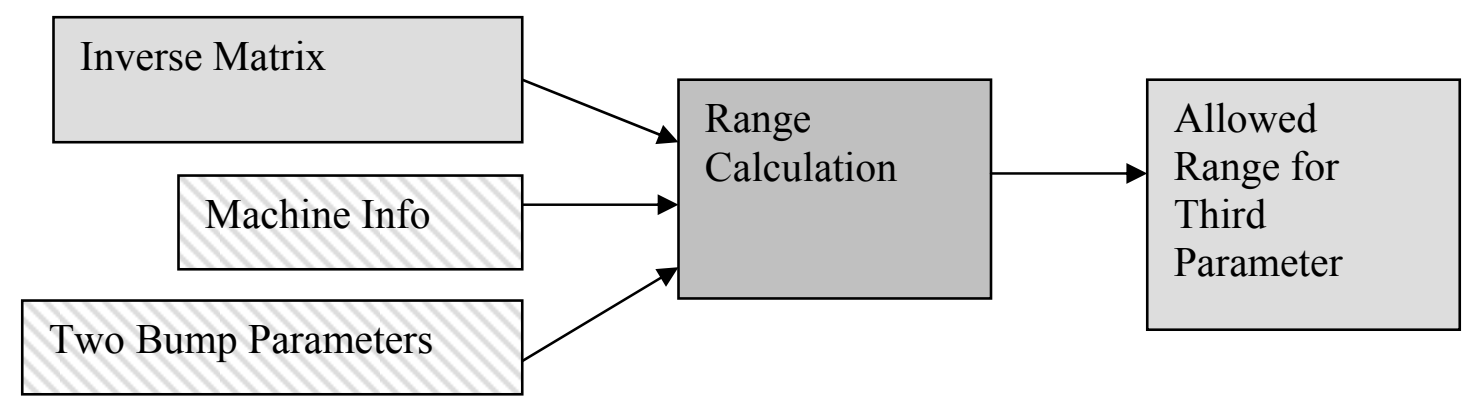

The range calculation will take the inverse matrix calculated above, the machine info, two of the bump parameters, and calculate the allowed range for the third parameter. 
This range will be displayed to the user in the first window. If the user specifies only one offset and then presses calculate, they will use the preset angle to find the range of the third. If the user sets two offsets and then presses "Change Value x'[D3]", this calculation will output the allowed range for the angle, given the two offsets.

The discussion in section III above gives us a basic scheme for calculating the range: Test the sign of $A_{i j}{ }^{-1}$ for each "i", populate a list of left limits and of right limits, and then return the maximum of the left limits and the minimum of the right limits. This technique can easily be performed using the following routine:

Define three equations for D3prime, D3, and D6 which correspond to equations (37), (38), and (39). The input for each of these three equations will be the index of the desired winding, the current in that winding, the other two parameters, the rigidity, the length of the magnets, the winding factor, and the main dipole current. The appropriate matrix entries should be able to be called from memory.

Let: $\mathrm{L}=\{$ right limits $\}$ empty at the beginning $\mathrm{J}=\{$ left limits $\}$ empty at the beginning

For clarity we will call $\mathrm{x}_{\mathrm{i}}(600)=$ the value of the variable parameter with $600 \mathrm{amps}$ in the $i^{\text {th }}$ winding, $x_{i}(-600)=$ the value of the variable parameter with -600 amps in the $i^{\text {th }}$ winding.

Determine the variable parameter. Use the appropriate function for the variable parameter:

For each winding, i:

Test the sign of $\mathrm{A}_{\mathrm{ij}}{ }^{-1}$.

If $\mathrm{A}_{\mathrm{ij}}{ }^{-1}$ positive, then add the value of $\mathrm{x}_{\mathrm{i}}(600)$ to $\mathrm{J}$ and $\mathrm{x}_{\mathrm{i}}(-600)$,

If $A_{i j}{ }^{-1}$ is negative add $x_{i}(600)$ to $L$ and $x(-600)$ to $J$.

Find the max of $\mathrm{J}$ and the min of $\mathrm{L}$. The range will be from the max of $\mathrm{J}$ to the $\min$ of $\mathrm{L}$. If $\max (\mathrm{J})>\min (\mathrm{L})$ then there is no allowed range.

Refer to section III to see how this method can be implemented in Mathematica.

\section{$\underline{\text { Reducing Currents }}$}

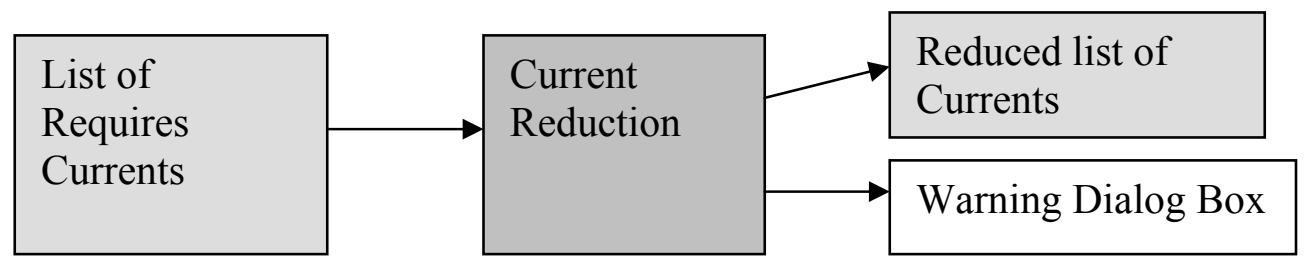

The current reduction calculation takes the required list of currents and test if any power supplies exceed their limit. It then produces a new list of currents where all values that exceed the limit are changed to the limit of the same sign. This calculation will be preformed in both the first window and the second window, and each time it's performed, a dialog box will be produced if any power supplies are requested to exceed their limit. 
In the first window, the output displayed is still the list of required currents. However, in the second window, the output displayed is the list of reduced currents.

The following is a simple way to test the list of currents and change any that are over 600 amps back to 600 amps. Here one inputs the list of currents and the reduced list, called "currents", is returned.

Let currents $=\{$ list of reduced currents $\}$ be empty at the beginning.

Then, for each current, test if its absolute value is greater than 600 .

If not, then simply add that value to "currents."

If it is, then add the value "sign $\left(\mathrm{I}_{\mathrm{i}}\right) 600$ " to the "currents."

It's important that the order of the list be preserved so that IC7 is 1 , ID1 is 2 , ID4 is 3, ID7 is 4 and IE1 is 5, since that is the way the transfer matrix has been defined.

The production of the dialog box should be equally routine.

\section{$\underline{\text { Kick Calculation }}$}

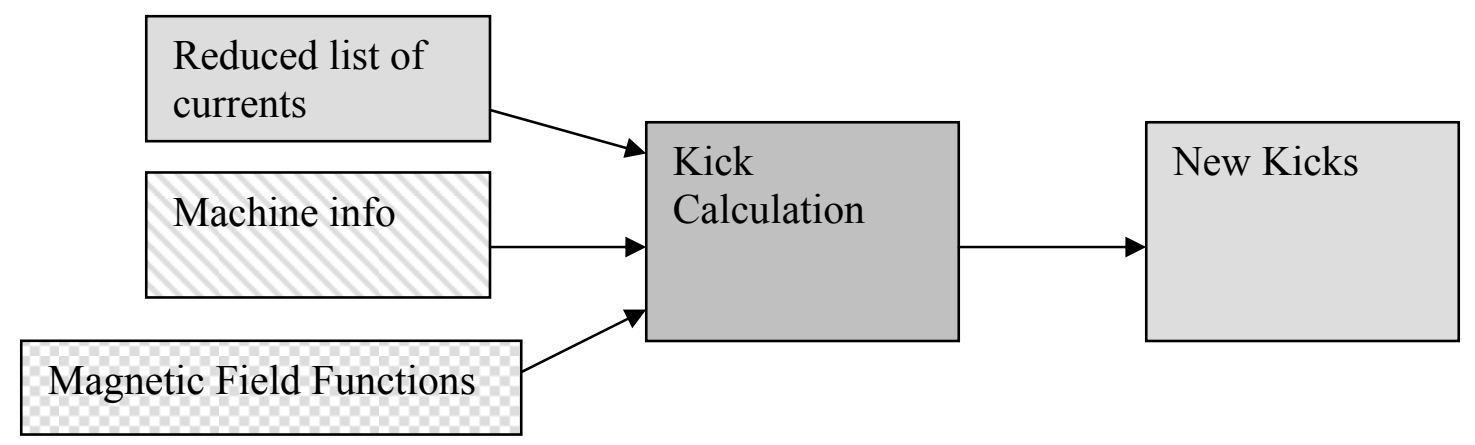

The kick calculation takes the reduced list of currents and the machine info and uses the magnetic field functions to output the kicks associated with these currents. The new kicks will not be shown to the user but will be internally stored to be used for other calculations.

Since both the amplitude of the residuals and the new offsets are calculated from the kicks we find these separately. To do this; first, find the new change in magnetic field needed using (41), and then, find the new kick using equation (42). These two steps can be easily combined to find the new kicks directly from the new currents using equation (43). These five kicks should be combined into a vector that can be multiplied by the transfer matrix.

\section{$\underline{\text { New Offset Calculation }}$}

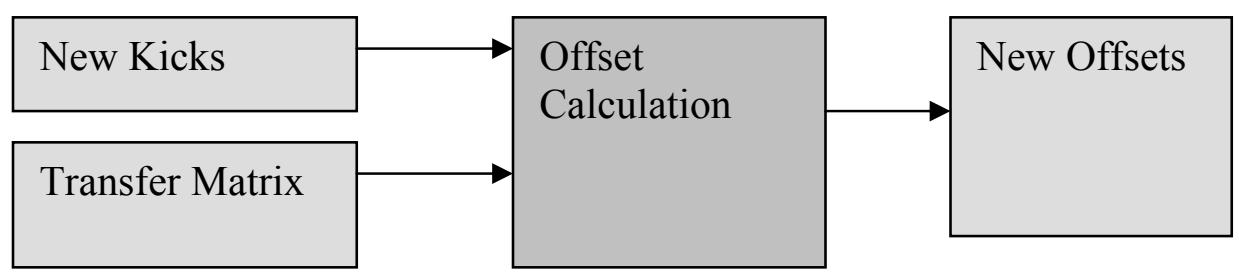


To find the new offsets, take the list of kicks (treating it like a vector), and multiply it by the transfer matrix, as outlined in equation (44). The output will be the list of offsets in the order, $x[\mathrm{E} 1], \mathrm{x}^{\prime}[\mathrm{E} 1], \mathrm{x}[\mathrm{D} 3], \mathrm{x}^{\prime}[\mathrm{D} 3]$, and $\mathrm{x}[\mathrm{D} 6]$.

Amplitude of the Residuals

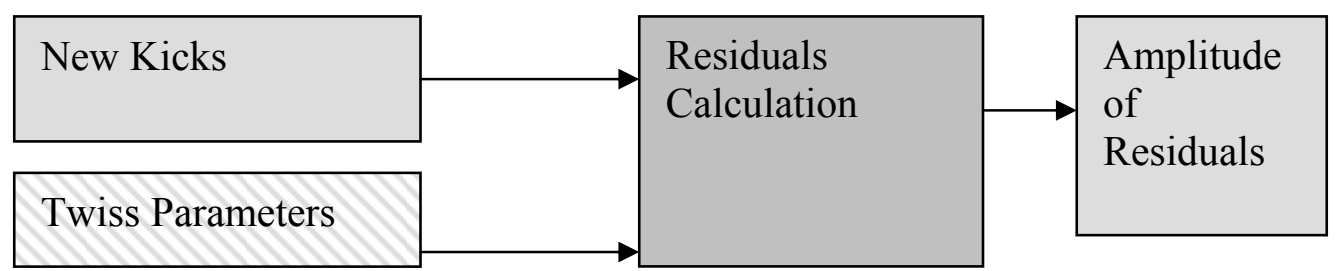

This is a simple matter of entering the twiss parameters at each of the windings (the value of $\beta$ and the phase advance $\varphi$, the same as used to evaluate the transfer matrix), the new kicks at each winding $\theta_{\mathrm{i}}$, the tune $v$, and the maximum of the beta function $\beta_{\mathrm{m}}$, into equation (45). Summing over the two indices "i" and " $j$ " independently we get the maximum amplitude of the residuals.

\section{Acknowledgements}

I would like to acknowledge Joseph M. Brennan, Michael Blaskiewicz, Jorg Kewisch, Kevin Brown, Todd Satogata, John Laster, and Lee Hammons for their support and aid in this project. 


\section{Appendix}

\section{$\underline{\text { Transfer Matrix }}$}

The following details the transfer matrix needed in the calculations regarding the NSRL extraction bump. It is important to note that the kicks associated with the trim windings have a conventional order in the kick vector, which creates an index convention for all values associated with that trim winding. The convention is the following: 1 is $\mathrm{C} 7$, 2 is D1, 3 is D4, 4 is D7, and 5 is E1. This convention will be used to denote values for the twiss parameters below.

Following is each row of the transfer matrix given using the index convention explained above. The index "i" in each of these equations will be run from 1 to 5 , creating 5 different matrix elements.

\section{First Row:}

$$
A_{1 i}=\frac{\sqrt{\beta(E 1)}}{2 \sin (\pi v)} \sqrt{\beta_{i}} \cos \left(\left|\phi(E 1)-\phi_{i}\right|-\pi v\right)
$$

Second Row:

$$
A_{2 i}=-\frac{\alpha(E 1)}{\beta(E 1)}\left(\frac{\sqrt{\beta(E 1)}}{2 \sin (\pi v)} \sqrt{\beta_{i}} \cos \left(\left|\phi(E 1)-\phi_{i}\right|-\pi v\right)\right)-\frac{1}{2 \sin (\pi v) \sqrt{\beta(E 1)}} \sqrt{\beta_{i}} \sin \left(\left|\phi(E 1)-\phi_{i}\right|-\pi v\right)
$$

Third Row:

$$
A_{3 i}=\frac{\sqrt{\beta(D 3)}}{2 \sin (\pi v)} \sqrt{\beta_{i}} \cos \left(\left|\phi(D 3)-\phi_{i}\right|-\pi v\right)
$$

Fourth Row:

$$
A_{4 i}=-\frac{\alpha(D 3)}{\beta(D 3)}\left(\frac{\sqrt{\beta(D 3)}}{2 \sin (\pi v)} \sqrt{\beta_{i}} \cos \left(\left|\phi(D 3)-\phi_{i}\right|-\pi v\right)\right)-\frac{1}{2 \sin (\pi v) \sqrt{\beta(D 3)}} \sqrt{\beta_{i}} \sin \left(\left|\phi(D 3)-\phi_{i}\right|-\pi v\right)
$$

\section{Fifth Row:}

$$
A_{5 i}=\frac{\sqrt{\beta(D 6)}}{2 \sin (\pi v)} \sqrt{\beta_{i}} \cos \left(\left|\phi(D 6)-\phi_{i}\right|-\pi v\right)
$$

